\title{
Tropical Theta Functions and Riemann-Roch Inequality for Tropical Abelian Surfaces
}

\author{
Ken Sumi
}

June 23, 2020

\begin{abstract}
We show that the space of theta functions on tropical tori is identified with a convex polyhedron. We also show a Riemann-Roch inequality for tropical abelian surfaces by calculating the self-intersection numbers of divisors.
\end{abstract}

\section{Introduction}

Tropical geometry is a field of mathematics studying piecewise-linear objects that appear as certain degenerate limits of algebraic varieties. Several results in algebraic geometry have analogies in tropical geometry. One of the famous analogies is a Riemann-Roch theorem for compact tropical curves.

A Riemann-Roch theorem for finite graphs was discovered by Baker-Norine [3] in 2007 and extended to tropical curves by Gathmann-Kerber [8] and MikhalkinZharkov 16] in 2008. The tropical Riemann-Roch theorem states that, for a compact tropical curve $C$ of genus $g$ and a tropical divisor $D$ on $C$, we have

$$
r(D)-r(K-D)=\operatorname{deg} D-g+1 .
$$

The number $r(D)$ is a substitute for the dimension of the complete linear system $|D|=\mathbb{P}\left(H^{0}(C, \mathcal{O}(D))\right)$, called the rank of the divisor $D$. In general, however, $r(D)$ is different from the dimension of $|D|$ as a polyhedral complex.

It is a very interesting problem to generalize the tropical Riemann-Roch theorem to higher dimensions. A main obstacle to higher dimensional generalization is to define the Euler characteristic $\chi(X, \mathcal{O}(D))$ : firstly the higher cohomology $H^{i}(X, \mathcal{O}(D))$ with $i \geq 1$ is not defined in tropical geometry; secondly the dimension of the space $\Gamma(X, \mathcal{O}(D))$ of global sections of $\mathcal{O}(D)$ can be different from the number $r(D)+1$ appearing in the Riemann-Roch theorem.

Tropical versions of Noether's formula were shown by Cartwright [5] for weak tropical surfaces and by Shaw 123 for compact tropical surface in the sense of Mikhalkin. They can be viewed as a Riemann-Roch theorem for the trivial divisor on a tropical surface. They avoid the above problems by considering the topological Euler characteristic of tropical surfaces. 
Cartwright conjectured a Riemann-Roch inequality for weak tropical surfaces in [6, Conjecture 3.6]. He introduced a higher dimensional analogue $h^{0}(X, D)$ of $r(D)+1$ and proposed a Riemann-Roch inequality by omitting $h^{1}(X, D)$ and assuming the Serre duality i.e. replacing $h^{2}(X, D)$ with $h^{0}(X, K-$ $D)$, where $K$ is the canonical divisor.

In this paper, we show the Cartwright conjecture for tropical abelian varieties, that is,

Theorem 1 (Corollary 18). Let $X$ be a tropical abelian surface and let $D$ be a divisor on $X$. Then the following inequality holds;

$$
h^{0}(X, D)+h^{0}(X,-D) \geq \frac{1}{2} D^{2} .
$$

A relation between $h^{0}(X, D)$ and the topology of the set of regular section $\Gamma(X, \mathcal{O}(D))$ is also an interesting question. We obtained the following results.

Theorem 2 (Theorem 39). Let $X=\mathbb{R}^{n} / \Lambda$ be a tropical torus and let $L \rightarrow X$ be a tropical line bundle. If $\Gamma(X, L) \neq\{-\infty\}, \Gamma(X, L)$ is identified with a convex polyhedron and $\mathbb{P}(\Gamma(X, L))$ is identified with a compact convex polyhedron; in particular it is pure-dimensional.

Theorem 3 (Theorem 44). Let $X$ be a tropical torus and let $D$ be a divisor on $X$. Then $h^{0}(X, D)$ defined by Cartwright [6] coincides with the dimension of $\Gamma(X, \mathcal{O}(D))$ as a convex polyhedron.

If a tropical analogue of Hirzebruch-Riemann-Roch theorem holds for $n$ dimensional tropical abelian varieties, then $\frac{1}{n !} D^{n}$ should be integral. In the last section, we show this.

Proposition 4 (Theorem 47). For a divisor $D$ on a tropical abelian variety, $\frac{1}{n !} D^{n}$ is an integer.

After the first version of the paper was put on the arXiv, Dustin Cartwright kindly suggested to the author that statements as in Theorem 3 and Theorem 47 may hold. We would like to thank him for his valuable suggestions.

\section{Tropical modules}

The tropical semifield $\mathbb{T}$ is the set $\mathbb{R} \cup\{-\infty\}$ equipped with the tropical sum $" x+y "=\max \{x, y\}$ and the tropical product " $x y "=x+y$ for $x, y \in \mathbb{T}$. The set of invertible elements of $\mathbb{T}$ is $\mathbb{R}$. A commutative semigroup $V$ equipped with the unit $\{-\infty\}$ and a scalar product $\mathbb{T} \times V \rightarrow V$ is called a tropical module or a $\mathbb{T}$-module if the following conditions are satisfied:

- " $(x+y) v "=" x v+y v "$ for any $x, y \in \mathbb{T}$, any $v \in V$;

- " $x(v+w) "=$ " $x v+x w "$ for any $x \in \mathbb{T}$, any $v, w \in V$; 
- " $x(y v) "=$ " $(x y) v "$ for any $x, y \in \mathbb{T}$, any $v \in V$;

- " $0 \cdot v "=v$ for the multiplicative unit $0 \in \mathbb{T}$ and any $v \in V$;

- if " $x v "=$ " $y v$ " for some $x, y \in \mathbb{T}$ and $v \in \mathbb{T}$, then $x=y$ or $v=-\infty$.

By these conditions, we have " $-\infty \cdot v "=-\infty$. For example, $\mathbb{T}^{n}$ is naturally a $\mathbb{T}$-module. Let $e_{i} \in \mathbb{T}^{n}$ be the element with 0 in the $i$ th coordinate and $-\infty$ in the other coordinates.

Let $V, W$ be tropical modules. A map $f: V \rightarrow W$ is called a tropical linear morphism if $f$ satisfies $f\left(" v_{1}+v_{2} "\right)=" f\left(v_{1}\right)+f\left(v_{2}\right) "$ and $f(" t v ")=" t f(v) "$ for any $v, v_{1}, v_{2} \in V, t \in \mathbb{T}$. If there is the inverse $f^{-1}$ and this is also a tropical linear morphism, then we call $f$ an isomorphism.

Let $V$ be a tropical module. Its projectivization $\mathbb{P}(V)$ is the quotient of $V \backslash\{-\infty\}$ by the equivalence relation $\sim$ where $v \sim v^{\prime}$ for $v, v^{\prime} \in V$ if there exists $t \in \mathbb{R}$ such that $v^{\prime}=$ " $t v "$.

Example 5. Let $V_{n}$ be the tropical submodule of $\mathbb{T}^{n}$ generated by

$$
f_{1}:=(-\infty, 0, \ldots, 0), f_{2}:=(0,-\infty, 0, \ldots, 0), \ldots, f_{n}:=(0, \ldots, 0,-\infty) .
$$

For any linear combination $a_{1} f_{1}+a_{2} f_{2}+\cdots+a_{n} f_{n}$ with $a_{i} \geq a_{j} \geq$ (the others), $a_{1} f_{1}+a_{2} f_{2}+\cdots+a_{n} f_{n}$ is the element whose $i$ th coordinate is $a_{j}$ and the other coordinates are $a_{i}$. For example, if $a_{1} \geq a_{2} \geq a_{k}, k=3, \ldots, n$, we get $a_{1} f_{1}+a_{2} f_{2}+\cdots+a_{n} f_{n}=\left(a_{2}, a_{1}, \ldots, a_{1}\right)$.

The projectivization $\mathbb{P}\left(V_{n}\right) \subset \mathbb{P}\left(\mathbb{T}^{n}\right)$ is the finite graph with $n+1$ vertices $[0, \ldots, 0],[-\infty, 0, \ldots, 0], \ldots,[0, \ldots, 0,-\infty]$ such that the valency of the central vertex $[0, \ldots, 0]$ is $n$ and the valency of the other vertex is 1 .

Example 6. A function $f: \mathbb{R}^{n} \rightarrow \mathbb{T}$ is a tropical polynomial (resp. tropical Laurent polynomial) if $f$ is a constant map to $\{-\infty\}$ or is of the form $f(x)=$ $\max _{j \in S}\left\{a_{j}+j \cdot x\right\}$, where $S$ is a finite subset of $\left(\mathbb{Z}_{\geq 0}\right)^{n}\left(\right.$ resp. $\left.\mathbb{Z}^{n}\right)$ and $a_{j} \in \mathbb{R}$. A tropical polynomial naturally extends to a function from $\mathbb{T}^{n}$ to $\mathbb{T}$. The set of tropical polynomials on $\mathbb{R}^{n}$ is naturally a tropical module.

Clearly, a tropical Laurent monomial is a $\mathbb{Z}$-affine linear function, that is, an affine linear function on $\mathbb{R}^{n}$ whose slope is in $\left(\mathbb{Z}^{n}\right)^{*}$.

\section{Tropical Tori}

The goal of this section is to show the Theorem 2.

\subsection{Tropical manifold and line bundles}

In this section we briefly introduce some basic notions of tropical geometry and give some examples. References are made to Allermann-Rau [1], Mikhalkin [15] and Mikhalkin-Zharkov [16], [17]. 
Definition 7. Let $U, V$ be open subsets of $\mathbb{R}^{n}, \mathbb{R}^{m}$ respectively. A map $f$ : $U \rightarrow V$ is $\mathbb{Z}$-affine linear if $f$ is given by a restriction of an affine map whose linear part is represented by an integral matrix.

Definition 8. [10, Definition 1.22] A tropical (or integral affine) manifold $M$ is a topological manifold with an atlas $\left\{\left(U_{i}, \psi_{i}\right)\right\}$ such that $\psi_{i} \circ \psi_{j}^{-1}$ is a restriction of a $\mathbb{Z}$-affine linear map on $\mathbb{R}^{n}$ for each $U_{i} \cap U_{j} \neq \emptyset$, where $n=\operatorname{dim} M$.

A map $f: M_{1} \rightarrow M_{2}$ between tropical manifolds is tropical if it is locally written as a $\mathbb{Z}$-affine map.

Remark 9. In the literature, tropical manifolds usually mean more general spaces, see Mikhalkin-Zharkov [17, Definition 1.14]. We adopt the above definition (following [10, Definition 1.22]) since we are only interested in tropical tori in this paper.

Example 10. The Euclidean space $\mathbb{R}^{n}$ is a tropical manifold.

Example 11. Fix the standard lattice $\mathbb{Z}^{n} \subset \mathbb{R}^{n}$. Let $\Lambda \subset \mathbb{R}^{n}$ be a lattice, that is, a discrete additive abelian subgroup of $\mathbb{R}^{n}$ of rank $n$. The quotient $\mathbb{R}^{n} / \Lambda$ is called a tropical torus. This is a tropical manifold whose integral structure is given by the standard lattice $\mathbb{Z}^{n}$.

Definition 12. Let $U$ be an open subset of a tropical manifold $M$. A continuous function $f: U \rightarrow \mathbb{T}$ is said to be regular if $f$ is locally the restriction of a tropical Laurent polynomial on $\mathbb{R}^{n}$.

A continuous function $h: U \rightarrow \mathbb{T}$ is said to be rational if for all $x \in U$ there exists an open neighborhood $V \subset U$ of $x$ and two regular functions $f, g$ on $V$ such that $\left.h\right|_{V}=" \frac{f}{g} "=f-g$.

The structure sheaf $\mathcal{O}_{U}$ of $U$ is the sheaf of regular functions on $U$. The sheaf $\mathcal{O}_{U}^{*}$ is the subsheaf of $\mathcal{O}_{U}$ consisting of invertible regular functions, that is, $\mathbb{Z}$-affine linear functions. We denote $\mathcal{O}^{*}$ by $\operatorname{Aff}_{\mathbb{Z}}(X)$. This sheaf is locally constant.

Remark 13. Since the structure sheaf $\mathcal{O}_{X}$ is NOT an abelian sheaf, we cannot consider the $i$ th Čech cohomology group $H^{i}\left(X, \mathcal{O}_{X}\right)$ for $i>0$. But since $\mathcal{O}_{X}^{*}$ is a multiplicative abelian sheaf, we can consider $H^{1}\left(X, \mathcal{O}_{X}^{*}\right)$ as in the classical case.

Definition 14. 2, Definitions 1.5] Let $M$ be an $n$-dimensional tropical manifold. A tropical line bundle on $M$ is a tuple $\left(L, \pi,\left\{U_{i}, \Psi_{i}\right\}_{i}\right)$ of a topological space $L$, a continuous surjection $\pi: L \rightarrow M$, an open covering $\left\{U_{i}\right\}$ called the trivializing covering and homeomorphisms $\Psi_{i}: \pi^{-1}\left(U_{i}\right) \cong U_{i} \times \mathbb{T}$ called trivializations which satisfy:

- The following diagram is commute:

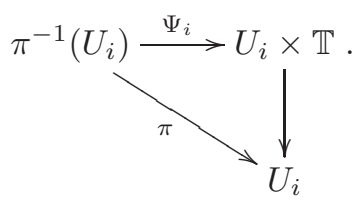


Here the vertical map is the first projection;

- For every $i, j$ with $U_{i} \cap U_{j} \neq \emptyset$, there exists a $\mathbb{Z}$-affine linear function $\varphi_{i j}: U_{i} \cap U_{j} \rightarrow \mathbb{R}$ such that $\Psi_{j} \circ \Psi_{i}^{-1}:\left(U_{i} \cap U_{j}\right) \times \mathbb{T} \rightarrow\left(U_{i} \cap U_{j}\right) \times \mathbb{T}$ is given by $(x, t) \mapsto\left(x, " \varphi_{i j}(x) t\right.$ "). These $\varphi_{i j}$ are called a transition functions of $L$.

It is clear that transition functions $\varphi_{i j}$ satisfies the cocycle condition $\varphi_{i j} \circ \varphi_{j k}=$ $\varphi_{i k}$. We identify two tropical line bundles $\left(L, \pi,\left\{U_{i}, \Psi_{i}\right\}_{i \in I}\right)$ and $\left(L, \pi,\left\{U_{j}, \Psi_{j}\right\}_{j \in J}\right)$ if $\left(L, \pi,\left\{U_{i}, \Psi_{i}\right\}_{i \in I \sqcup J}\right)$ is a tropical line bundle on $M$, that is, satisfies the second condition above. We often write $L$ for $\left(L, \pi,\left\{U_{i}, \Psi_{i}\right\}_{i}\right)$ to avoid heavy notation.

We can refine the trivializing covering for $L$ as follows. If a covering $\left\{V_{j}\right\}$ is a refinement of the trivializing covering $\left\{U_{i}\right\}$ for $L$, then the trivializations $\Psi_{i}$ over $U_{i}$ induce trivializations $\Phi_{j}$ over $V_{j}$ and the line bundles $\left(L, \pi,\left\{U_{i}, \Psi_{i}\right\}\right)$ and $\left(L, \pi,\left\{V_{i}, \Phi_{i}\right\}\right)$ are identified.

Given an open covering $\left\{U_{i}\right\}$ of $M$ and transition functions $\varphi_{i j}: U_{i} \cap U_{j} \rightarrow \mathbb{R}$ satisfying the cocycle condition, we can construct a line bundle $L$ by $L=\coprod_{i} U_{i} \times$ $\mathbb{T} / \sim$. Here $\left(x_{i}, t_{i}\right) \sim\left(x_{j}, t_{j}\right)$ for $\left(x_{i}, t_{i}\right) \in U_{i} \times \mathbb{T}$ and $\left(x_{j}, t_{j}\right) \in U_{j} \times \mathbb{T}$ means $x_{i}=x_{j} \in U_{i} \cap U_{j}$ and $t_{j}=$ " $\varphi_{i j}(x) t_{i} "$.

Definition 15. [2, Lemma 1.16] Let $\left(L_{1}, \pi_{1},\left\{U_{1 i}, \Psi_{1 i}\right\}\right),\left(L_{2}, \pi_{2},\left\{U_{2 j}, \Psi_{2 j}\right\}\right)$ be two tropical line bundles on a tropical manifold $M$. By taking a common refinement of the trivializing coverings $\left\{U_{1 i}\right\},\left\{U_{2 i}\right\}$ if necessary, we may assume that $U_{1 i}=U_{2 i}=: U_{i}$. Then $L_{1}$ and $L_{2}$ are said to be isomorphic, denoted by $L_{1} \cong L_{2}$, if there exist a map $f: L_{1} \rightarrow L_{2}$ and invertible regular functions $h_{i j}: U_{i} \cap U_{j} \rightarrow \mathbb{T}$ such that $\Psi_{2 j} \circ f \circ \Psi_{1 i}^{-1}:\left(U_{i} \cap U_{j}\right) \times \mathbb{T} \rightarrow\left(U_{i} \cap U_{j}\right) \times \mathbb{T}$ is of the form $(x, t) \mapsto\left(x, " h_{i j}(x) t "\right)$,

We can see that $L_{1}$ and $L_{2}$ are isomorphic if and only if there exist an invertible regular function $g_{i}: U_{i} \rightarrow \mathbb{R}$ for each $i$ such that " $g_{i}(x) \varphi_{i j}^{1}(x) g_{j}(x)^{-1}$ " = $\varphi_{i j}^{2}(x)$ for each $U_{i} \cap U_{j} \neq \emptyset$. Here $\varphi_{i j}^{1}, \varphi_{i j}^{2}$ are the transition function of $L_{1}, L_{2}$ respectively.

Definition 16. We take two line bundles $L_{1}$ and $L_{2}$ as above. The tensor product $L_{1} \otimes L_{2}$ is defined to be the tropical line bundle on $M$ whose transition functions are " $\varphi_{i j}^{1} \varphi_{i j}^{2} "=\varphi_{i j}^{1}+\varphi_{i j}^{2}$.

The inverse $L_{1}^{-1}$ of the line bundle $L_{1}$ is defined to be the tropical line bundle on $M$ whose transition functions are " $\left(\varphi_{i j}^{1}\right)^{-1 "}=-\varphi_{i j}^{1}$.

Definition 17. Let $\pi: L \rightarrow M$ be a line bundle on a tropical manifold $M$ and $U$ be an open subset of $M$. A function $s: U \rightarrow \pi^{-1}(U)$ is a regular section (resp. rational section) on $U$ of $L$ if $\pi \circ s$ is the identity map on $U$ and $p_{i} \circ \Psi_{i} \circ s$ is a regular (resp. rational) function, where $p_{i}: U_{i} \times \mathbb{T} \rightarrow \mathbb{T}$ is the second projection. If $U=M$ then we call $s$ a global regular (resp. rational) section or simply a regular (resp. rational) section. The set $\Gamma(U, L)$ of regular sections on $U$ of $L$ naturally has the structure of a tropical module.

The tropical Picard group $\operatorname{Pic}(M)$ is the set of isomorphic classes of tropical line bundles on $M$. The product and inverse are given by the tensor product 
and the inverse of line bundles, respectively. It is clear that $\operatorname{Pic}(M)$ is naturally identified with $H^{1}\left(M, \mathcal{O}_{M}^{*}\right)=H^{1}\left(M, \mathrm{Aff}_{\mathbb{Z}}\right)$ via transition functions.

We can also define the pull-back of line bundles as in the classical case. Let $f: M \rightarrow M^{\prime}$ be a tropical map between tropical manifolds and let $\pi: L \rightarrow M^{\prime}$ be a tropical line bundle with the trivializing covering $\left\{U_{i}\right\}$ and the transition functions $\varphi_{i j}$. Then we define the pull-back as

$$
f^{*} L:=\{(x, \xi) \in M \times L \mid f(x)=\pi(\xi)\}
$$

with the trivializing covering is $\left\{f^{-1}\left(U_{i}\right)\right\}$ and the transition functions are $\varphi_{i j} \circ f$.

\subsection{Divisors on tropical tori}

In this section, we briefly introduce the notion of divisors. For the details of divisors, we refer the reader to Allermann-Rau [1] or Mikhalkin [15].

Definition 18. A rational polyhedron in $\mathbb{R}^{n}$ is a finite intersection of closed half spaces with rational slopes, that is, a subset of the form $\bigcap_{i \in I}\left\{x \in \mathbb{R}^{n} \mid a_{i} \cdot x \geq b_{i}\right\}$ where $I$ is a finite set and $a_{i} \in \mathbb{Z}^{n}, b_{i} \in \mathbb{R}$. A face of a rational polyhedron $\sigma$ is a subset of the form $\sigma \cap\left\{x \in \mathbb{R}^{n} \mid a \cdot x=b\right\}$ with $a \in \mathbb{Z}^{n}$ and $b \in \mathbb{R}$ satisfying $\sigma \subset\left\{x \in \mathbb{R}^{n} \mid a \cdot x \geq b\right\}$

Let $M$ be a tropical manifold with an atlas $\left\{\left(U_{i}, \psi_{i}\right)\right\}$. A rational polyhedron in a tropical manifold $M$ is a subset $\sigma \subset M$ such that $\psi_{i}\left(\sigma \cap U_{i}\right)$ is an intersection of $\operatorname{Im} \psi_{i}$ and a rational polyhedron $\rho_{i}$ in $\mathbb{R}^{n}$ for each $i$. A face of $\sigma$ is a subset $\tau \subset \sigma$ such that $\psi_{i}\left(\tau \cap U_{i}\right)$ is an intersection of $\operatorname{Im} \psi_{i}$ and a face of $\rho_{i}$ in $\mathbb{R}^{n}$ for each $i$.

Definition 19. A rational polyhedral complex on a tropical manifold $M$ is a locally finite collection $\Sigma$ of rational polyhedra in $M$ satisfying the following:

- Any face of $\sigma \in \Sigma$ belongs to $\Sigma$;

- For $\sigma, \tau \in \Sigma$ with $\sigma \cap \tau \neq \emptyset$, the intersection is a face of both $\sigma$ and $\tau$.

Polyhedra belonging to $\Sigma$ are called cells. A rational polyhedral complex is said to be pure $k$ dimensional if its maximal cells (with respect to inclusions) have dimension $k$.

Definition 20. Let $M$ be an $n$ dimensional tropical manifold. A weight of a pure $n-1$ dimensional polyhedral complex $\Sigma$ on $M$ is a function $w$ from the set of $n-1$ cells of $\Sigma$ to $\mathbb{Z}$. Then we call the pair $(\Sigma, w)$ a weighted rational polyhedral complex.

Definition 21. Let $(\Sigma, w)$ be a pure $n-1$ dimensional weighted polyhedral complex on an open subset of $\mathbb{R}^{n}$. Fix an $n-2$ dimensional cell $P$ of $\Sigma$ and let $F_{1}, \ldots, F_{k}$ be the $n-1$ dimensional cells adjacent to $P$. We define $L(P) \subset \mathbb{R}^{n}$ to be the tangent space of $P$. Let $v_{1}, \ldots, v_{k} \in \mathbb{Z}^{n} / L(P) \cap \mathbb{Z}^{n}$ be the primitive outgoing vectors from the origin parallel to $F_{1} / L(P), \ldots, F_{k} / L(P)$ respectively. 
Then $(\Sigma, w)$ is said to be balanced at $P$ if $\sum_{i=1, \ldots, k} w\left(F_{i}\right) v_{i}=0$ and $(\Sigma, w)$ is said to be balanced if it is balanced at all $n-2$ dimensional faces $P$ of $\Sigma$.

Let $M$ be a tropical manifold with an atlas $\left\{\left(U_{i}, \psi_{i}\right)\right\}$ and let $\left(\Sigma^{\prime}, w^{\prime}\right)$ be a pure $n-1$ dimensional weighted polyhedral complex on $M$. $\left(\Sigma^{\prime}, w^{\prime}\right)$ is said to be balanced if its restriction to $U_{i}$ is identified with a balanced weighted polyhedral complex via $\psi_{i}$.

Example 22. Let $f: \mathbb{R}^{n} \rightarrow \mathbb{R}$ be a piecewise-linear function whose slopes lie in $\left(\mathbb{Z}^{n}\right)^{*}$ and $D(f)$ be the locus where $f$ is nonlinear on any neighborhood. Then $D(f)$ is a (non-compact) polyhedral complex whose maximal cells have integral slopes.

For each maximal cell of $D(f)$, we can define the multiplicity given by $f$ as follows. For each maximal cell $F$ of $D(f)$, there exist two connected components $V_{1}, V_{2}$ of $\mathbb{R}^{n}-D(f)$ on which the restriction $\left.f\right|_{V_{i}}$ is affine linear and the intersection of the closures of $V_{1}$ and $V_{2}$ is $F$. Then the difference of the slopes of $\left.f\right|_{V_{1}},\left.f\right|_{V_{2}}$ is in $\left(\mathbb{Z}^{n}\right)^{*}$. Thus we can take the lattice length $l_{F}$ of this difference, that is, the maximal positive integer $k$ such that this difference can be divided by $k$. We define the multiplicity of $F$ as the lattice length $l_{F}$ if $f$ is convex around $F$, as $-l_{F}$ if $f$ is concave around $F$. Then $D(f)$ is not only a weighted integral polyhedral complex, but also a balanced one.

We draw some examples of balanced weighted graph on $\mathbb{R}^{2}$ given by $f_{1}=$ $\max \{x, y, 0\}, f_{2}=\max \{2 x, y, x+y, 0\}$ and $f_{3}=\max \{3 x, 3 y, 2 x+y, 0\}$ in Figure 田, 2 and 3 .

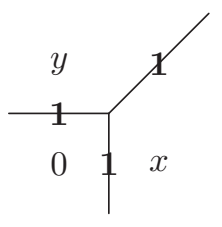

Figure 1: $D\left(f_{1}(x, y)\right)$

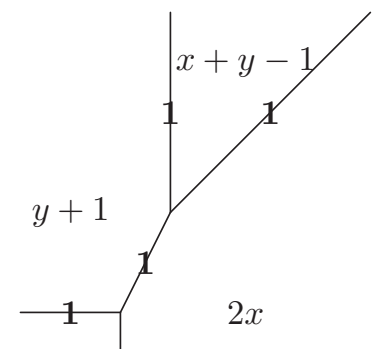

0

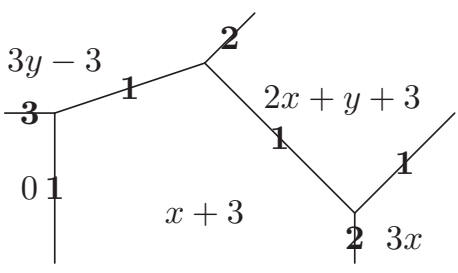

Figure 3: $D\left(f_{3}(x, y)\right)$

Figure 2: $D\left(f_{2}(x, y)\right)$

Definition 23. [15, Definition 4.10] Let $X=\mathbb{R}^{n} / \Lambda$ be an $n$-dimensional tropical torus.

A divisor $D$ on $X$ is a balanced weighted polyhedral complex on $X$. Any divisor can be locally written as $D(f)$ for a rational function $f$ on an open subset of $X$. In other words, a divisor is given by data $\left\{\left(U_{i}, f_{i}\right)\right\}$ where $\left\{U_{i}\right\}$ is a covering of $X$ and $\left\{f_{i}: U_{i} \rightarrow \mathbb{R}\right\}$ is a rational function such that $f_{i}-f_{j}$ is a $\mathbb{Z}$-affine linear function on $U_{i} \cap U_{j}$.

We identify two divisors if they become the same after subdivision and deletion of cells of weight zero. The set of divisors on $X$ is called the divisor group 
of $X$, denoted $\operatorname{Div}(X)$. Here the sum of divisors on $X$ is defined by a common subdivision and the sum of weight functions (restricted to the subdivision), and the inverse of a divisor is defined by a divisor whose support is the original support and whose weight function is -1 times the original weight.

In terms of local data, the sum of divisors $\left\{\left(U_{i}, f_{i}\right)\right\}$ and $\left\{\left(V_{i}, g_{i}\right)\right\}$ is given by $\left\{\left(U_{i} \cap V_{j}, f_{i}+g_{j}\right)\right\}$ and the inverse of a divisor $\left\{\left(U_{i}, f_{i}\right)\right\}$ is given by $\left\{\left(U_{i},-f_{i}\right)\right\}$.

As in the classical case, divisors give rise to line bundles. Let $D$ be a divisor on $X$ and $\left\{\left(U_{i}, f_{i}\right)\right\}$ be the local data of $D$. Then we can define the line bundle $\mathcal{O}(D)$ associated to $D$ to be the line bundle on $X$ whose transition functions are given by $\varphi_{i j}=f_{i}-f_{j}$. It is clear that the above map from the divisor group to the Picard group is a group homomorphism.

Two divisors $D_{1}, D_{2}$ on $X$ are linearly equivalent if the difference $D_{1}-D_{2}$ is given by a global rational function, or in other words, $\mathcal{O}\left(D_{1}\right)$ and $\mathcal{O}\left(D_{2}\right)$ are isomorphic.

\subsection{Theta functions}

The content of this section is basically due to Mikhalkin-Zharkov [16].

Let $X=\mathbb{R}^{n} / \Lambda$ be a tropical torus. The sheaf $\mathcal{T}_{\mathbb{Z}}^{*}$ is defined by the following exact sequence of sheaves of abelian groups:

$$
0 \rightarrow \underline{\mathbb{R}} \rightarrow \mathrm{Aff}_{\mathbb{Z}} \rightarrow \mathcal{T}_{\mathbb{Z}}^{*} \rightarrow 0
$$

where $\underline{\mathbb{R}}$ denote the constant sheaf on $X$ whose stalks are $\mathbb{R}$. Then $\mathcal{T}_{\mathbb{Z}}^{*} \cong \underline{\left(\mathbb{Z}^{n}\right)^{*}}$.

Taking the first sheaf cohomology, we get the Chern class map

$$
c_{1}: H^{1}\left(X, \operatorname{Aff}_{\mathbb{Z}}\right) \rightarrow H^{1}\left(X, \mathcal{T}_{\mathbb{Z}}^{*}\right) .
$$

We obtain the following proposition by analyzing the coboundary map of the long exact sequence.

Proposition 24 (Mikhalkin-Zharkov [16] Section 5.1). The image of $c_{1}$ in $H^{1}\left(X, \mathcal{T}_{\mathbb{Z}}^{*}\right) \cong \Lambda^{*} \otimes\left(\mathbb{Z}^{n}\right)^{*}$ is the set of elements of $\Lambda^{*} \otimes\left(\mathbb{Z}^{n}\right)^{*}$ which can be naturally extended to symmetric bilinear forms on $\mathbb{R}^{n}$. Here $\Lambda^{*}:=\operatorname{Hom}_{\mathbb{Z}}(\Lambda, \mathbb{Z})$.

Definition 25. If a class $[c] \in \operatorname{Im} c_{1}$ is positive definite as a symmetric form on $\mathbb{R}^{n}$, it is called a polarization. The degree of a polarization is the cardinal of the cokernel $\operatorname{Cok}([c])=\left(\mathbb{Z}^{n}\right)^{*} /[c](\Lambda)$, where we identify $[c] \in \Lambda^{*} \otimes\left(\mathbb{Z}^{n}\right)^{*}$ with the corresponding element of $\operatorname{Hom}_{\mathbb{Z}}\left(\Lambda,\left(\mathbb{Z}^{n}\right)^{*}\right)$. If a polarization $[c] \in \operatorname{Hom}_{\mathbb{Z}}\left(\Lambda,\left(\mathbb{Z}^{n}\right)^{*}\right)$ is an isomorphism, $[c]$ is said to be principal.

Definition 26. A tropical abelian variety is a tropical torus which has a polarization. A pair $(X,[c])$ of tropical abelian variety and its polarization is called polarized tropical abelian variety.

In this section we consider tropical tori, which are not necessarily tropical abelian varieties. We will consider abelian surfaces to calculate the intersection numbers in Section 0 . 
Let $X=\mathbb{R} / \Lambda$ be a tropical torus and let $L$ be a tropical line bundle on $X$. We set $Q:=c_{1}(L) \in \Lambda^{*} \otimes\left(\mathbb{Z}^{n}\right)^{*}$.

Since the sheaf of invertible regular functions $\mathrm{Aff}_{\mathbb{Z}}$ is constant on $\mathbb{R}^{n}$, the first cohomology $H^{1}\left(\mathbb{R}^{n}, \mathrm{Aff}_{\mathbb{Z}}\right)$ vanishes. Thus the pull back $p^{*} L$ of $L$ by the projection $p: \mathbb{R}^{n} \rightarrow \mathbb{R}^{n} / \Lambda$ is isomorphic to the trivial bundle $\mathbb{R}^{n} \times \mathbb{T}$. Fix this isomorphism once.

The lattice $\Lambda$ acts on $\mathbb{R}^{n}$ by translation. We lift this action to an action on $p^{*} L$ by

$$
\lambda \cdot(x, \xi):=(x+\lambda, \xi), \lambda \in \Lambda, x \in \mathbb{R}^{n}, \xi \in L .
$$

By the isomorphism $p^{*} L \cong \mathbb{R}^{n} \times \mathbb{T}$ we get a $\Lambda$-action on $\mathbb{R}^{n} \times \mathbb{T}$. This action can be described as

$$
\lambda \cdot(x, t):=\left(x+\lambda, t+Q_{\mathbb{R}}(\lambda, x)+\beta_{L}(\lambda)\right)
$$

for some $\beta_{L}: \Lambda \rightarrow \mathbb{R}$. Here $Q_{\mathbb{R}} \in\left(\mathbb{R}^{n}\right)^{*} \otimes\left(\mathbb{R}^{n}\right)^{*}$ is the extension of $Q$ on $\mathbb{R}^{n}$. By the associativity of the action, $\beta_{L}$ satisfies $\beta_{L}\left(\lambda_{1}+\lambda_{2}\right)=\beta_{L}\left(\lambda_{1}\right)+\beta_{L}\left(\lambda_{2}\right)+$ $Q_{\mathbb{R}}\left(\lambda_{1}, \lambda_{2}\right)$ for any $\lambda_{1}, \lambda_{2} \in \Lambda$. Thus $\beta_{L}(\lambda)-\frac{1}{2} Q_{\mathbb{R}}(\lambda, \lambda)$ is linear in $\lambda$. We denote this linear function by $\alpha_{L}(\lambda)$.

Definition 27. For a linear function $\alpha: \mathbb{R}^{n} \rightarrow \mathbb{R}$ and $Q \in(\Lambda)^{*} \otimes\left(\mathbb{Z}^{n}\right)^{*}$ which extends to a symmetric form on $\mathbb{R}^{n}$, We define a $\Lambda$-action on $\mathbb{R}^{n} \times \mathbb{T}$ by

$$
\lambda \cdot(x, t):=\left(x+\lambda, t+Q_{\mathbb{R}}(\lambda, x)+\frac{1}{2} Q_{\mathbb{R}}(\lambda, \lambda)+\alpha(\lambda)\right)
$$

for all $\lambda \in \Lambda, x \in \mathbb{R}^{n}, t \in \mathbb{T}$. We can easily check that this defines a $\Lambda$-action. Then we define the line bundle $L(Q, \alpha)$ on $X=\mathbb{R}^{n} / \Lambda$ by $\left(\mathbb{R}^{n} \times \mathbb{T}\right) / \Lambda$. The local trivializations of $L(Q, \alpha)$ are induced from the given trivialization of the trivial bundle $\mathbb{R}^{n} \times \mathbb{T}$.

Now we get the following proposition.

Proposition 28. The map $L(\cdot, \cdot): \operatorname{Im} c_{1} \times\left(\mathbb{R}^{n}\right)^{*} \rightarrow H^{1}(X, \mathrm{Aff} \mathbb{Z})$ given by $(Q, \alpha) \mapsto L(Q, \alpha)$ is a surjective group homomorphism. Moreover, the kernel of this homomorphism is $0 \times\left(\mathbb{Z}^{n}\right)^{*}$, that is, $L(Q, \alpha) \cong L(Q, \alpha+\gamma)$ for any $\gamma \in\left(\mathbb{Z}^{n}\right)^{*}$.

Proof. The long exact sequence given by (1) induces the following short exact sequence;

$$
0 \rightarrow H^{1}(X, \underline{\mathbb{R}}) / H^{0}\left(X, \mathcal{T}_{\mathbb{Z}}^{*}\right)=\left(\mathbb{R}^{n}\right)^{*} /\left(\mathbb{Z}^{n}\right)^{*} \rightarrow H^{1}\left(X, \mathrm{Aff}_{\mathbb{Z}}\right) \rightarrow \operatorname{Im} c_{1} \rightarrow 0 .
$$

This splits by $L(\cdot, 0): \operatorname{Im} c_{1} \rightarrow H^{1}\left(X, \mathrm{Aff}_{\mathbb{Z}}\right)$, thus $H^{1}\left(X, \mathrm{Aff}_{\mathbb{Z}}\right)$ is isomorphic to $\operatorname{Im} c_{1} \times\left(\left(\mathbb{R}^{n}\right)^{*} /\left(\mathbb{Z}^{n}\right)^{*}\right)$. Then the map $L(\cdot, \cdot)$ is identified with the quotient map $\operatorname{Im} c_{1} \times\left(\mathbb{R}^{n}\right)^{*} \rightarrow \operatorname{Im} c_{1} \times\left(\left(\mathbb{R}^{n}\right)^{*} /\left(\mathbb{Z}^{n}\right)^{*}\right)$. The kernel of this homomorphism is clearly $0 \times\left(\mathbb{Z}^{n}\right)^{*}$. 
Any global section of $L(Q, \alpha)$ is identified with a regular function $\Theta: \mathbb{R}^{n} \rightarrow \mathbb{R}$ satisfying the following quasi-periodicity condition:

$$
\Theta(x+\lambda)=\Theta(x)+Q_{\mathbb{R}}(\lambda, x)+\beta(\lambda), \lambda \in \Lambda, x \in \mathbb{R}^{n}
$$

with $\beta(\lambda)$ is equal to $\alpha(\lambda)+\frac{1}{2} Q(\lambda, \lambda)$. The regularity implies that $\Theta$ is convex and the quasi-periodicity implies the fact that if $b \in\left(\mathbb{Z}^{n}\right)^{*}$ is a slope of $\Theta$, then $b+Q(\lambda, \cdot)$ is also a slope of $\Theta$ for any $\lambda \in \Lambda$.

By the above argument, the tropical module $\Gamma(X, L(Q, \alpha))$ of regular sections of $L(Q, \alpha)$ is identified with the set

$$
\left\{\begin{array}{l|l}
\Theta: \mathbb{R}^{n} \rightarrow \mathbb{R} & \begin{array}{l}
\Theta(x+\lambda)=\Theta(x)+Q_{\mathbb{R}}(\lambda, x)+\beta(\lambda), \lambda \in \Lambda, x \in \mathbb{R}^{n} \\
\Theta(x) \text { is a regular function. }
\end{array}
\end{array}\right\} \cup\{-\infty\} .
$$

An element of this set which is not $\{-\infty\}$ is called a (tropical) theta function.

Remark 29. If $Q$ has a negative eigenvalue, $\beta(\lambda)$ diverges to $-\infty$ quadratically in the direction of the eigenvector. Then any function satisfying the quasiperiodicity is not convex, thus no theta function exists

For $r \in \mathbb{R}^{n}$, we define the translation $\iota_{r}: \mathbb{R}^{n} / \Lambda \rightarrow \mathbb{R}^{n} / \Lambda$ by $x \mapsto x+r$. The pulled back line bundle $\iota_{r}^{*} L(Q, \alpha)$ is given by the quotient of $\mathbb{R}^{n} \times \mathbb{T}$ by the following $\Lambda$-action.

$$
\lambda \cdot(x-r, t):=\left(x-r+\lambda, t+Q_{\mathbb{R}}(\lambda, x)+\frac{1}{2} Q_{\mathbb{R}}(\lambda, \lambda)+\alpha(\lambda)\right) .
$$

That is,

$$
\begin{aligned}
\lambda \cdot(x, t) & =\left(x+\lambda, t+Q_{\mathbb{R}}(\lambda, x+r)+\frac{1}{2} Q_{\mathbb{R}}(\lambda, \lambda)+\alpha(\lambda)\right) \\
& =\left(x+\lambda, t+Q_{\mathbb{R}}(\lambda, x)+\frac{1}{2} Q_{\mathbb{R}}(\lambda, \lambda)+\alpha(\lambda)+Q_{\mathbb{R}}(\lambda, r)\right) .
\end{aligned}
$$

Thus we get $\iota_{r}^{*} L(Q, \alpha)=L\left(Q, \alpha+Q_{\mathbb{R}}(\cdot, r)\right)$.

\subsection{The space of theta function: positive definite case}

Given a bilinear form $Q \in \Lambda^{*} \otimes\left(\mathbb{Z}^{n}\right)^{*}$, let $q$ denote the linear map $\Lambda \rightarrow\left(\mathbb{Z}^{n}\right)^{*}$, $\lambda \mapsto Q(\lambda, \cdot)$ and let $q_{\mathbb{R}}$ denote the linear map $\mathbb{R}^{n} \rightarrow\left(\mathbb{R}^{n}\right)^{*}$ extending $q$.

In this section, we consider the case where $Q$ is positive definite as a symmetric bilinear form. Then for any $\alpha \in\left(\mathbb{R}^{n}\right)^{*}$, there exists an element $r \in \mathbb{R}^{n}$ such that $\alpha=q_{\mathbb{R}}(r)$.

Let $\Theta$ be a theta function for $L\left(Q, q_{\mathbb{R}}(r)\right)$ and consider the Legendre transform of $\Theta$,

$$
\widehat{\Theta}(a)=\max _{x \in \mathbb{R}^{n}}\{a \cdot x-\Theta(x)\}, a \in\left(\mathbb{Z}^{n}\right)^{*} .
$$

The maximum exists since $\Theta$ diverges to $+\infty$ quadratically as $|x| \rightarrow \infty$; this follows by the positive definiteness of $Q$ and quasi-periodicity of $\Theta$. The Legendre 
transform $\widehat{\Theta}:\left(\mathbb{Z}^{n}\right)^{*} \rightarrow \mathbb{R}$ is convex, that is, $\widehat{\Theta}(a) \leq \sum t_{i} \widehat{\Theta}\left(a_{i}\right)$ for $a, a_{i} \in\left(\mathbb{Z}^{n}\right)^{*}$ and $0 \leq t_{i} \leq 1$ satisfying $\sum t_{i}=1, a=\sum t_{i} a_{i}$.

The Legendre transform $\widehat{\Theta}$ satisfies the following quasi-periodicity:

$$
\begin{aligned}
\widehat{\Theta}(a+q(-\lambda)) & =\max _{x \in \mathbb{R}^{n}}\{(a+q(-\lambda)) \cdot x-\Theta(x)\} \\
& =\max _{x \in \mathbb{R}^{n}}\left\{a \cdot x-\Theta(x+\lambda)+\frac{1}{2} Q_{\mathbb{R}}(\lambda, \lambda)+q_{\mathbb{R}}(r)(\lambda)\right\} \\
& =\max _{x \in \mathbb{R}^{n}}\left\{a \cdot(x-\lambda)-\Theta(x)+\frac{1}{2} Q_{\mathbb{R}}(\lambda, \lambda)+q_{\mathbb{R}}(r)(\lambda)\right\} \\
& =\max _{x \in \mathbb{R}^{n}}\{a \cdot x-\Theta(x)\}-a \cdot \lambda+\frac{1}{2} Q_{\mathbb{R}}(\lambda, \lambda)+Q_{\mathbb{R}}(\lambda, r) \\
& =\widehat{\Theta}(a)-a \cdot \lambda+\frac{1}{2} Q_{\mathbb{R}}(\lambda, \lambda)+Q_{\mathbb{R}}(\lambda, r)
\end{aligned}
$$

for all $a \in\left(\mathbb{Z}^{n}\right)^{*}, \lambda \in \Lambda$. Here the second equality follows by the quasiperiodicity of $\Theta$ and the third equality follows by replacing $x$ with $x-\lambda$. Moreover, the Legendre transformation gives one-to-one correspondence between the set of theta functions for $L\left(Q, q_{\mathbb{R}}(r)\right)$ and the following set:

$$
\left\{\begin{array}{l|l}
\eta:\left(\mathbb{Z}^{n}\right)^{*} \rightarrow \mathbb{R} & \begin{array}{l}
\eta \text { is convex and for all } \lambda \in \Lambda, a \in\left(\mathbb{Z}^{n}\right)^{*}, \\
\eta(a+q(\lambda))=\eta(a)+a \cdot \lambda+\frac{1}{2} Q_{\mathbb{R}}(\lambda, \lambda)-Q_{\mathbb{R}}(r, \lambda)
\end{array}
\end{array}\right\} .
$$

We choose a subset $B \subset\left(\mathbb{Z}^{n}\right)^{*}$ such that the natural map $B \rightarrow\left(\mathbb{Z}^{n}\right)^{*} / q(\Lambda)$ is a bijection. Then $\widehat{\Theta}$ is determined by the values at $b \in B$ by the quasiperiodicity. Because of the convexity of the theta function $\Theta$, the inverse Legendre transform of $\widehat{\Theta}$ is $\Theta$. Thus $\Theta(x)$ can be written as follows:

$$
\begin{aligned}
\Theta(x) & =\max _{a \in\left(\mathbb{Z}^{n}\right)^{*}}\{a \cdot x-\widehat{\Theta}(a)\} \\
& =\max _{b \in B} \max _{\lambda \in \Lambda}\{(b+q(-\lambda)) \cdot x-\widehat{\Theta}(b+q(-\lambda))\} \\
& =\max _{b \in B} \max _{\lambda \in \Lambda}\left\{(b+q(-\lambda)) \cdot x-\widehat{\Theta}(b)+b \cdot \lambda-\frac{1}{2} Q_{\mathbb{R}}(\lambda, \lambda)-Q_{\mathbb{R}}(\lambda, r)\right\} \\
& =\max _{b \in B}\left\{\Theta_{b}(x)+\frac{1}{2} Q_{\mathbb{R}}\left(q_{\mathbb{R}}^{-1}(b)-r, q_{\mathbb{R}}^{-1}(b)-r\right)-\widehat{\Theta}(b)\right\},
\end{aligned}
$$

where

$$
\Theta_{b}(x):=\max _{\lambda \in \Lambda}\left\{(b+q(\lambda)) \cdot x-\frac{1}{2} Q_{\mathbb{R}}\left(\lambda+q_{\mathbb{R}}^{-1}(b)-r, \lambda+q_{\mathbb{R}}^{-1}(b)-r\right)\right\} .
$$

By the above argument, we get the fact that theta functions for $L\left(Q, q_{\mathbb{R}}(r)\right)$ exist and every theta function for $L\left(Q, q_{\mathbb{R}}(r)\right)$ can be written in the form:

$$
\max _{b \in B}\left\{\Theta_{b}(x)+s_{b}\right\}
$$


for some $\left\{s_{b}\right\} \in \mathbb{T}^{B}$. The set of slopes of $\Theta_{b}$ is $b+q(\Lambda)$ and the sets $b+q(\Lambda), b \in B$ are pairwise disjoint. Thus $\Theta_{b}$ cannot be written as any tropical linear combination of elements of $H^{0}\left(X, L\left(Q, q_{\mathbb{R}}(r)\right)\right) \backslash \mathbb{T} \Theta_{b}$. Consequently any minimal generating set of the $\mathbb{T}$-module $\Gamma\left(X, L\left(Q, q_{\mathbb{R}}(r)\right)\right)$ is of the form $\left\{\Theta_{b}+s_{b}\right\}_{b \in B}$; in particular, the minimal number of generators is $|B|$. Let $\pi: \mathbb{T}^{B} \rightarrow H^{0}\left(X, L\left(Q, q_{\mathbb{R}}(r)\right)\right)$ be the surjective tropical homomorphism $\left(s_{b}\right)_{b \in B} \mapsto \max _{b \in B}\left\{\Theta_{b}+s_{b}\right\}$.

Definition 30. We define the map $\varphi: \Gamma\left(X, L\left(Q, q_{\mathbb{R}}(r)\right)\right) \rightarrow \mathbb{T}^{B}$ by

$$
\varphi(\Theta)=\left(\varphi^{b}(\Theta)\right)_{b \in B}
$$

where

$$
\begin{aligned}
\varphi^{b}(\Theta) & :=\max \left\{s_{b} \in \mathbb{T} \mid \Theta(x) \geq \Theta_{b}(x)+s_{b} \forall x \in \mathbb{R}^{n}\right\} \\
& =\min _{x \in \mathbb{R}^{n}}\left\{\Theta(x)-\Theta_{b}(x)\right\}
\end{aligned}
$$

The composition $\pi \circ \varphi$ is the identity map of $\Gamma\left(X, L\left(Q, q_{\mathbb{R}}(r)\right)\right)$. Thus the map $\varphi$ is injective and we can induce a topology of $\Gamma\left(X, L\left(Q, q_{\mathbb{R}}(r)\right)\right)$ by identifying it with the subspace $\varphi\left(\Gamma\left(X, L\left(Q, q_{\mathbb{R}}(r)\right)\right)\right) \subset \mathbb{T}^{B}$.

Remark 31. The map $\varphi: \Gamma\left(X, L\left(Q, q_{\mathbb{R}}(r)\right)\right) \rightarrow \mathbb{T}^{B}$ is not a map of tropical modules. This map measures the "meaningful" coefficients $s_{b}$ of $\Theta_{b}$ appearing in the expression $\Theta(x)=\max _{b \in B}\left\{\Theta_{b}(x)+s_{b}\right\}$.

Proposition 32. Let $r(b)$ denote $\frac{1}{2} Q_{\mathbb{R}}\left(q_{\mathbb{R}}^{-1}(b)-r, q_{\mathbb{R}}^{-1}(b)-r\right)$. Then

$$
\varphi^{b}(\Theta)=-\widehat{\Theta}(b)+r(b)
$$

Proof. Recall the set of slopes of a generator

$$
\Theta_{b}(x)=\max _{\lambda \in \Lambda}\left\{(b+q(\lambda)) \cdot x-\frac{1}{2} Q_{\mathbb{R}}\left(\lambda+q_{\mathbb{R}}^{-1}(b)-r, \lambda+q_{\mathbb{R}}^{-1}(b)-r\right)\right\}
$$

is exactly $\{b+q(\lambda) \mid \lambda \in \Lambda\}$. Let $D_{\lambda}^{b}$ be the maximal closed domain on which $\Theta_{b}(x)$ is an affine linear function of slope $b+q(\lambda)$. By the quasi-periodicity, we get

$$
D_{\lambda_{1}+\lambda_{2}}^{b}=\lambda_{2}+D_{\lambda_{1}}^{b}
$$

for $\lambda_{1}, \lambda_{2} \in \Lambda$ and thus the image of $D_{\lambda}^{b}$ by the quotient map $\mathbb{R}^{n} \rightarrow X=\mathbb{R}^{n} / \Lambda$ is the whole of $X$. It is clear that $\Theta_{b}(x)=b \cdot x-r(b)$ holds for any point $x$ in the fundamental domain $D_{0}^{b}$ of $X$. 
Since $\Theta(x)-\Theta_{b}(x)$ is $\Lambda$-periodic by the quasi-periodicity of $\Theta(x)$,

$$
\begin{aligned}
\varphi^{b}(\Theta) & =\min _{x \in \mathbb{R}^{n}}\left\{\Theta(x)-\Theta_{b}(x)\right\} \\
& =\min _{x \in D_{0}^{b}}\left\{\Theta(x)-\Theta_{b}(x)\right\} \\
& =\min _{x \in D_{0}^{b}}\{\Theta(x)-(b \cdot x-r(b))\} \\
& \left.=\min _{x \in \mathbb{R}^{n}}\{\Theta(x)-(b \cdot x-r(b))\} \quad \text { (by the convexity of } \Theta_{b}\right) \\
& =-\max _{x \in \mathbb{R}^{n}}\{b \cdot x-\Theta(x)\}+r(b) \\
& =-\widehat{\Theta}(b)+r(b) .
\end{aligned}
$$

Example 33. Let us consider a tropical torus $\mathbb{R} / \mathbb{Z}$ and a line bundle $L(3,0)$. We take $B=\{0,1,2\}$ and consider the generators of $\Gamma(\mathbb{R} / \mathbb{Z}, L(3,0))$,

$$
\Theta_{b}(x)=\max _{n \in \mathbb{Z}}\left\{(3 n+b) x-\frac{3}{2}\left(\frac{3 n+b}{3}\right)^{2}\right\}, b \in B .
$$

Let $\varphi: \Gamma(\mathbb{R} / \mathbb{Z}, L(3,0)) \rightarrow \mathbb{T}^{3}$ be the injection as in Definition 30. Let us calculate the set $\{(0, r, s) \mid r, s \in \mathbb{R}\} \cap \varphi(\Gamma(\mathbb{R} / \mathbb{Z}, L(3,0)))$; this is identified with the tropical projectivization of $\varphi(\Gamma(\mathbb{R} / \mathbb{Z}, L(3,0)))$.

The strategy of calculation is as follows. First we calculate the region of possible values of $\varphi^{1}(\Theta)$ under the condition that $\varphi^{0}(\Theta)=0$. We can easily see that $\varphi^{1}(\Theta)$ ranges over a closed interval whose lower or upper bounds are given by the minimum or maximal of the function:

$$
\Theta_{0}(x)-\Theta_{1}(x)
$$

By computing these values, we get the range of $\varphi^{1}(\Theta)$. It is $\left[-\frac{1}{3}, \frac{1}{3}\right]$.

Next, we fix the value of $\varphi^{1}(\Theta)$ and denote the value by $r$. We calculate the upper and lower bound of $\varphi^{2}(\Theta)$. The lower bound is the minimal value of the difference of theta functions

$$
\max \left\{\Theta_{0}(x), \Theta_{1}(x)+r\right\}-\Theta_{2}(x) .
$$

The upper bound is the supremum of $s$ such that the terms $\Theta_{0}$ and $\Theta_{1}+r$ cannot be removed from the equation $\Theta=\max \left(\Theta_{0}, \Theta_{1}+r, \Theta_{2}+s\right)$, that is, the maximal $s$ satisfying the following two equations:

$$
\begin{aligned}
& 0 \geq \min _{x \in \mathbb{R}^{2}}\left\{\max \left\{\Theta_{1}(x)+r, \Theta_{2}(x)+s\right\}-\Theta_{0}(x)\right\} ; \\
& 0 \geq \min _{x \in \mathbb{R}^{2}}\left\{\max \left\{\Theta_{0}(x), \Theta_{2}(x)+s\right\}-\left(\Theta_{1}+r\right)\right\} .
\end{aligned}
$$


By computing these values, we get the range of $\varphi^{2}(\Theta)$. It is

$$
\frac{1}{2} r+\frac{1}{6} \leq \varphi^{2}(\Theta) \leq \min \{2 r,-r\}+\frac{1}{3} .
$$

Thus the slice $\{(0, r, s) \mid r, s \in \mathbb{R}\} \cap \varphi(\Gamma(\mathbb{R} / \mathbb{Z}, L(3,0)))$ is given by

$$
\left\{(r, s) \in \mathbb{R}^{2} \mid s \leq 2 r+\frac{1}{3}, r \leq 2 s+\frac{1}{3}, r+s \leq \frac{1}{3}\right\} .
$$

This is a 2-dimensional simplex as in Figure 4 and each of generators corresponds to a vertex of this simplex.

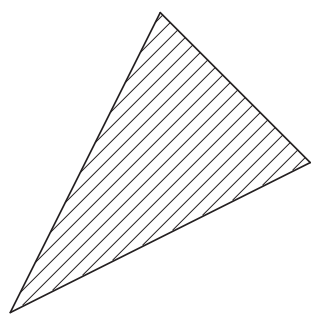

Figure 4: the slice $\{(0, r, s) \mid r, s \in \mathbb{R}\} \cap \varphi(\Gamma(\mathbb{R} / \mathbb{Z}, L(3,0)))$

Example 34. In the previous example, the projectivization of $\Gamma(X, L)$ is a simplex and its vertices correspond to generators. However it is not always true. We give a counter-example.

Let $q_{\mathbb{R}}: \mathbb{R}^{2} \rightarrow\left(\mathbb{R}^{2}\right)^{*}$ be the linear map given by $\left(\begin{array}{ll}2 & 1 \\ 1 & 2\end{array}\right)$ and let $\Lambda$ be the lattice $q_{\mathbb{R}}^{-1}\left(2\left(\mathbb{Z}^{2}\right)^{*}\right)$. These data define an element $Q \in \Lambda^{*} \otimes\left(\mathbb{Z}^{2}\right)^{*}$ given by $q_{\mathbb{R}}$ and a tropical abelian surface $X:=\left(\mathbb{R}^{2} / \Lambda, Q\right)$.

Let us consider the tropical line bundle $L=L(Q, 0)$ on $X$. We take $B=$ $\left\{b_{i j}\right\}_{i, j \in\{0,1\}}$ with $b_{i j}={ }^{t}(i, j)$ and consider the generators of $\Gamma(X, L)$,

$\Theta_{b_{i j}}(x)=\max _{k, l \in \mathbb{Z}}\left\{(2 k+i) x+(2 l+j) y-\frac{1}{3}\left((2 k+i)^{2}-(2 k+i)(2 l+j)+(2 l+j)^{2}\right)\right\}$.

Define the injection $\varphi: \Gamma(X, L) \rightarrow \mathbb{T}^{4}$ as

$$
\varphi(\Theta)=\left(\varphi^{00}(\Theta), \varphi^{01}(\Theta), \varphi^{10}(\Theta), \varphi^{11}(\Theta)\right)
$$

where $\varphi^{i j}(\Theta):=\varphi^{b_{i j}}(\Theta)$ is as in Definition 30. By computing as in the previous example, the slice $\{(0, r, s, t) \mid r, s, t \in \mathbb{R}\} \cap \varphi(\Gamma(X, L))$ is given by

$$
\left\{\begin{array}{l|l}
(0, r, s, t) & \begin{array}{l}
-\frac{1}{3} \leq r \leq \frac{1}{3}, \max \{0, r\}-\frac{1}{3} \leq s \leq \min \{0, r\}+\frac{1}{3}, \\
\max \{0, r, s\}-\frac{1}{3} \leq t \leq \min \{0, r, s\}+\frac{1}{3}
\end{array}
\end{array}\right\} .
$$


This region can also be written as

$$
\bigcup_{-\frac{1}{6} \leq a \leq \frac{1}{6}}\left\{(r, s, t)+(a, a, a)|| r\left|\leq \frac{1}{6},\right| s\left|\leq \frac{1}{6},\right| t \mid \leq \frac{1}{6},\right\} .
$$

This is the convex polyhedron that has 14 vertices as in Figure 5 .

The 4 generators of the tropical module $\Gamma(X, L)$ correspond to vertices of this polyhedron, but this polyhedron has more vertices.

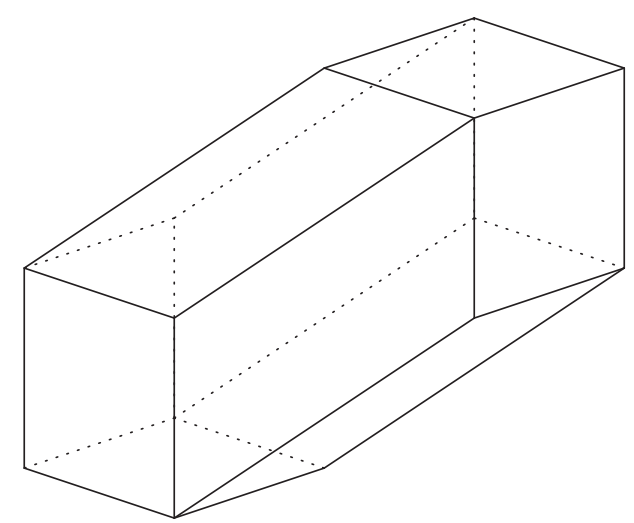

Figure 5: the slice $\{(0, r, s, t) \mid r, s, t \in \mathbb{R}\} \cap \varphi(\Gamma(X, L))$

Lemma 35. Let $D$ be a compact convex polyhedron in $\mathbb{R}^{n}$ and $f: D \times \mathbb{R}^{m} \rightarrow \mathbb{R}$ be a convex piecewise-linear function with finitely many slopes $\$$.

We define the function $g: \mathbb{R}^{m} \rightarrow \mathbb{R}$ as

$$
g(y):=\min _{x \in D}\{f(x, y)\} .
$$

Then $g$ is a convex piecewise-linear function with finitely many slopes.

Proof. Let $p: D \times \mathbb{R}^{n} \times \mathbb{R} \rightarrow \mathbb{R}^{n} \times \mathbb{R}$ be the projection and $\Delta_{f}$ be the upper convex hull of graph of $f$, that is,

$$
\Delta_{f}:=\left\{(x, y, r) \in D \times \mathbb{R}^{n} \times \mathbb{R} \mid r \geq f(x, y)\right\} .
$$

Since the set of slopes of $f$ is finite, $\Delta_{f}$ has only finitely many faces. Thus $\Delta_{f}$ is a convex polyhedron and the image of the projection $p\left(\Delta_{f}\right)$ is also a convex polyhedron. On the other hand $p\left(\Delta_{f}\right)$ is described as

$$
\begin{aligned}
p\left(\Delta_{f}\right) & =\left\{(y, r) \in \mathbb{R}^{n} \times \mathbb{R} \mid r \geq f(x, y) \text { for all } x \in D\right\} \\
& =\left\{(y, r) \in \mathbb{R}^{n} \times \mathbb{R} \mid r \geq \min _{x \in D}\{f(x, y)\}\right\} .
\end{aligned}
$$

\footnotetext{
${ }^{1} \mathrm{~A}$ convex piecewise-linear function means a function which is locally written as the maximum of finitely many affine linear functions. It has finitely many slopes if and only if it can be written as the maximum of finitely many affine linear functions.
} 
That is, $p\left(\Delta_{f}\right)$ is the upper convex hull of the graph of $g$. Therefore $g$ is a convex piecewise-linear function. Since the upper convex hull $p\left(\Delta_{f}\right)$ of $g$ has only finitely many faces, the convex piecewise-linear function $g$ has only finitely many slopes.

Theorem 36. Let $L$ be a tropical line bundle on a tropical abelian variety $X=\mathbb{R}^{n} / \Lambda$ such that $Q=c_{1}(L)$ is positive definite. Then $\Gamma(X, L)$ is generated by $|B|=\mid$ Cok $q \mid$ elements as a $\mathbb{T}$-module. Moreover, $\Gamma(X, L)$ is identified with an $|B|$-dimensional convex polyhedron in $\mathbb{T}^{B}$ via $\varphi$ of Definition 30 and its projectivization is identified with a compact polyhedron in $\mathbb{R}^{|B|-1}$.

Proof. We can assume $L=L\left(Q, q_{\mathbb{R}}(r)\right)$ for some $r \in \mathbb{R}^{*}$. The former is already shown. Thus we show the latter. The image $\operatorname{Im} \varphi$ is equal to the subset

$$
\left\{\left(t_{b}\right)_{b \in B} \mid \varphi^{b}\left(\max _{b^{\prime} \in B}\left\{\Theta_{b^{\prime}}(x)+t_{b^{\prime}}\right\}\right)=t_{b} \forall b \in B\right\} .
$$

The condition $\varphi^{b}\left(\max _{b^{\prime} \in B}\left\{\Theta_{b^{\prime}}(x)+t_{b^{\prime}}\right\}\right)=t_{b}$ is equivalent to

$$
\begin{aligned}
t_{b} & \geq \min _{x \in \mathbb{R}^{n}}\left\{\max _{b^{\prime} \in B \backslash\{b\}}\left(\Theta_{b^{\prime}}(x)+t_{b^{\prime}}\right)-\Theta_{b}(x)\right\} \\
& =\min _{x \in D_{0}^{b}}\left\{\max _{b^{\prime} \in B \backslash\{b\}}\left(\Theta_{b^{\prime}}(x)+t_{b^{\prime}}\right)-\Theta_{b}(x)\right\} \quad \text { (by the quasi-periodicity) } \\
& =\min _{x \in D_{0}^{b}}\left\{\max _{b^{\prime} \in B \backslash\{b\}}\left(\Theta_{b^{\prime}}(x)+t_{b^{\prime}}\right)-(b \cdot x-r(b))\right\},
\end{aligned}
$$

where $D_{0}^{b}$ is the region introduced in the proof of Proposition 32 .

By Lemma 35, the right-hand side of the above inequality is a convex piecewise-linear function of $t_{b^{\prime}}, b^{\prime} \in B \backslash\{b\}$ with only finitely many slopes. Thus $\operatorname{Im} \varphi$ is a convex polyhedron.

Let $\Xi:=\max _{b \in B}\left\{\Theta_{b}(x)\right\}$ and let $\widetilde{\Lambda} \subset \mathbb{R}^{n}$ be the lattice $q_{\mathbb{R}}^{-1}\left(\left(\mathbb{Z}^{2}\right)^{*}\right)$, which is an overlattice of $\Lambda$. We can check that $\Xi$ satisfies the quasi-periodicity

$$
\Xi(x+\eta)=\Xi(x)+Q_{\mathbb{R}}(\eta, x)+\frac{1}{2} Q_{\mathbb{R}}(\eta, \eta)+q_{\mathbb{R}}(r), \eta \in \widetilde{\Lambda}, x \in \mathbb{R}^{n}
$$

and we get that the set of slopes of $\Xi$ is exactly $\left(\mathbb{Z}^{n}\right)^{*}$. We can check that $\varphi(\Xi)=0$, thus the image $\operatorname{Im} \varphi$ contains the origin $(0, \ldots, 0)$. Moreover, the image $\operatorname{Im} \varphi$ contains $|B|$ points $(\varepsilon, 0, \ldots, 0), \ldots,(0, \ldots, 0, \varepsilon)$ for a sufficiently small positive number $\varepsilon$, for example,

$$
\varepsilon=\min _{b \in B}\left\{\min _{x \in \mathbb{R}^{n}}\left\{\max _{b^{\prime} \in B \backslash\{b\}}\left\{\Theta_{b^{\prime}}(x)\right\}-\Theta_{b}(x)\right\}\right\} .
$$

The convexity of $\operatorname{Im} \varphi$ tells us that $\operatorname{Im} \varphi$ includes an $|B|$-simplex. Therefore the image $\operatorname{Im} \varphi$ is an $|B|$-dimensional convex polyhedron. 
Remark 37. The convexity of $\varphi(\Gamma(X, L))$ is clear by Proposition 32 and the fact that the set (2) is convex.

Remark 38. Mikhalkin-Zharkov [16, Remark 5.5] states (without proof) that the dimension of $\Gamma(X, L)$ is given by the degree of the polarization. A novel observation in this paper is that $\Gamma(X, L)$ is a convex polyhedron.

\subsection{The space of theta function: positive semidefinite case}

Next, we consider a line bundle $L(Q, \alpha)$ on the tropical torus $X=\mathbb{R}^{n} / \Lambda$ with $Q$ positive semidefinite. Then $\operatorname{Ker}\left(q_{\mathbb{R}}\right)$ is not necessarily 0 and it is both $\Lambda$-rational and $\mathbb{Z}$-rational because of the symmetry of $Q \in \Lambda^{*} \otimes\left(\mathbb{Z}^{n}\right)^{*}$. Here a subspace of $\mathbb{R}^{n}$ is said to be $\Lambda$-rational (resp. $\mathbb{Z}$-rational) if it is generated by elements of $\Lambda$ $\left(\right.$ resp. $\left.\mathbb{Z}^{n}\right)$.

First, we suppose $\alpha \in \operatorname{Im}\left(q_{\mathbb{R}}\right)+\left(\mathbb{Z}^{n}\right)^{*}$. Then we can describe $\alpha=q_{\mathbb{R}}(r)+\gamma$ for some $r \in \mathbb{R}^{n}$ and $\gamma \in\left(\mathbb{Z}^{n}\right)^{*}$. Since $L(Q, \alpha) \cong L\left(Q, q_{\mathbb{R}}(r)\right)$, we can assume that $\alpha \in \operatorname{Im}\left(q_{\mathbb{R}}\right)$.

Recall the quasi-periodicity of theta functions for $L(Q, \alpha)$,

$$
\Theta(x+\lambda)=\Theta(x)+Q_{\mathbb{R}}(\lambda, x)+\frac{1}{2} Q_{\mathbb{R}}(\lambda, \lambda)+\alpha(\lambda) .
$$

By the quasi-periodicity, theta functions are $\operatorname{Ker}(q)$-periodic since $\alpha \in \operatorname{Im}\left(q_{\mathbb{R}}\right)$. Theta functions are convex and periodic in the direction $\operatorname{Ker}\left(q_{\mathbb{R}}\right)$, thus they are constant along $\operatorname{Ker}\left(q_{\mathbb{R}}\right)$, that is,

$$
\Theta\left(x+\lambda^{\prime \prime}\right)=\Theta(x) \text { for all } \lambda^{\prime \prime} \in \operatorname{Ker}\left(q_{\mathbb{R}}\right), x \in \mathbb{R}^{n} .
$$

Thus theta functions descends to functions on $\mathbb{R}^{n} / \operatorname{Ker}\left(q_{\mathbb{R}}\right)$. The quotient space $\mathbb{R}^{n} / \operatorname{Ker}\left(q_{\mathbb{R}}\right)$ contains the two natural lattices, $\mathbb{Z}^{n} /\left(\operatorname{Ker}\left(q_{\mathbb{R}}\right) \cap \mathbb{Z}^{n}\right)$ induced by $\mathbb{Z}^{n}$ and $\Lambda / \operatorname{Ker}(q)$ induced by $\Lambda$.

We denote a descendant theta function by $\bar{\Theta}$. This satisfies the following quasi-periodicity for all $x \in \mathbb{R}^{n} / \operatorname{Ker}\left(q_{\mathbb{R}}\right)$ and $\lambda \in \Lambda / \operatorname{Ker}(q)$;

$$
\bar{\Theta}(x+\lambda)=\bar{\Theta}(x)+\overline{Q_{\mathbb{R}}}(\lambda, x)+\frac{1}{2} \overline{Q_{\mathbb{R}}}(\lambda, \lambda)+\bar{\alpha}(\lambda) .
$$

Here $\overline{Q_{\mathbb{R}}} \in\left(\mathbb{R}^{n} / \operatorname{Ker}\left(q_{\mathbb{R}}\right)\right)^{*} \otimes\left(\mathbb{R}^{n} / \operatorname{Ker}\left(q_{\mathbb{R}}\right)\right)^{*}$ is the symmetric form induced by $Q$ and $\bar{\alpha}$ is the linear function on $\mathbb{R}^{n} / \operatorname{Ker}\left(q_{\mathbb{R}}\right)$ induced by $\alpha$.

By the above argument, the tropical module $\Gamma(X, L(Q, \alpha))$ is identified with

$$
\left\{\begin{array}{l|l}
\bar{\Theta}: \mathbb{R}^{n} / \operatorname{Ker}\left(q_{\mathbb{R}}\right) \rightarrow \mathbb{R} & \begin{array}{l}
\bar{\Theta}(x+\lambda)=\bar{\Theta}(x)+\overline{Q_{\mathbb{R}}}(\lambda, x)+\frac{1}{2} \overline{Q_{\mathbb{R}}}(\lambda, \lambda)+\bar{\alpha}(\lambda) \\
\text { for all } \lambda \in \Lambda / \operatorname{Ker}(q), x \in \mathbb{R}^{n} / \operatorname{Ker}\left(q_{\mathbb{R}}\right) . \\
\bar{\Theta} \text { is a }\left(\mathbb{Z}^{n} /\left(\operatorname{Ker}\left(q_{\mathbb{R}}\right) \cap \mathbb{Z}^{n}\right)\right)^{*} \text {-regular function. }
\end{array}
\end{array}\right\} \cup\{-\infty\} .
$$

Here a $\left(\mathbb{Z}^{n} /\left(\operatorname{Ker}\left(q_{\mathbb{R}}\right) \cap \mathbb{Z}^{n}\right)\right)^{*}$-regular function means a convex piecewise-linear function whose slopes lie in $\left(\mathbb{Z}^{n} /\left(\operatorname{Ker}\left(q_{\mathbb{R}}\right) \cap \mathbb{Z}^{n}\right)\right)^{*}$. 
Under the identification

$$
\begin{aligned}
\left(\mathbb{R}^{n} / \operatorname{Ker}\left(q_{\mathbb{R}}\right)\right)^{*} & =\left\{a \in\left(\mathbb{R}^{n}\right)^{*} \mid a\left(\operatorname{Ker}\left(q_{\mathbb{R}}\right)\right)=0\right\} \\
\left(\mathbb{Z}^{n} /\left(\operatorname{Ker}\left(q_{\mathbb{R}}\right) \cap \mathbb{Z}^{n}\right)\right)^{*} & =\left\{a \in\left(\mathbb{Z}^{n}\right)^{*} \mid a\left(\operatorname{Ker}\left(q_{\mathbb{R}}\right) \cap \mathbb{Z}^{n}\right)=0\right\},
\end{aligned}
$$

we have

$$
\begin{aligned}
\left(\mathbb{R}^{n} / \operatorname{Ker}\left(q_{\mathbb{R}}\right)\right)^{*} & =\operatorname{Im}\left(q_{\mathbb{R}}\right) \\
\left(\mathbb{Z}^{n} /\left(\operatorname{Ker}\left(q_{\mathbb{R}}\right) \cap \mathbb{Z}^{n}\right)\right)^{*} & =\operatorname{Im}\left(q_{\mathbb{R}}\right) \cap\left(\mathbb{Z}^{n}\right)^{*} \supset \operatorname{Im}(q)
\end{aligned}
$$

by the symmetry of $Q$.

In this situation, we can apply the result of the positive definite case. Let $\bar{q}: \Lambda / \operatorname{Ker}(q) \rightarrow \operatorname{Im}\left(q_{\mathbb{R}}\right) \cap\left(\mathbb{Z}^{n}\right)^{*}$ be the linear map given by $q$. Then we can see that $\Gamma(X, L)$ is generated by $l:=|\operatorname{Cok}(\bar{q})|=\mid$ the torsion part of $\operatorname{Cok}(q) \mid$ elements and that $\Gamma(X, L)$ naturally embeds into $\mathbb{T}^{l}$. Moreover, $\Gamma(X, L)$ has the structure of a pure $l$ dimensional polyhedron in $\mathbb{T}^{l}$, and its projectivization is a compact polyhedron.

Next, we suppose that $\alpha \notin \operatorname{Im}\left(q_{\mathbb{R}}\right)+\left(\mathbb{Z}^{n}\right)^{*}$. If $\alpha$ is integral on $\operatorname{Ker}\left(q_{\mathbb{R}}\right) \cap \mathbb{Z}^{n}$, that is, $\alpha\left(\operatorname{Ker}\left(q_{\mathbb{R}}\right) \cap \mathbb{Z}^{n}\right) \subset\left(\mathbb{Z}^{n}\right)^{*}$, we can assume that $\alpha\left(\operatorname{Ker}\left(q_{\mathbb{R}}\right) \cap \mathbb{Z}^{n}\right)=0$ by translating $\alpha$ by an element of $\left(\mathbb{Z}^{n}\right)^{*}$. Then we can consider $\alpha$ as a linear map from $\mathbb{R}^{n} / \operatorname{Ker}\left(q_{\mathbb{R}}\right)$ to $\mathbb{R}$, that is, $\alpha \in \operatorname{Im}\left(q_{\mathbb{R}}\right)$. This is a contradiction. Therefore, $\alpha$ is not integral even on $\operatorname{Ker}\left(q_{\mathbb{R}}\right) \cap \mathbb{Z}^{n}$.

By the quasi-periodicity, theta functions for $L(Q, \alpha)$ must satisfy

$$
\Theta\left(x+\lambda^{\prime \prime}\right)=\Theta(x)+\alpha\left(\lambda^{\prime \prime}\right) \text { for all } \lambda^{\prime \prime} \in \operatorname{Ker}\left(q_{\mathbb{R}}\right) \cap \Lambda, x \in \mathbb{R}^{n} .
$$

Thus theta functions are affine linear on $\operatorname{Ker}\left(q_{\mathbb{R}}\right)$ with slope $\alpha$. Since $\alpha$ is not integral on $\operatorname{Ker}\left(q_{\mathbb{R}}\right) \cap \mathbb{Z}^{n}$, theta functions cannot be regular. Thus $\Gamma(X, L)=$ $\{-\infty\}$.

Consequently we get the following theorem.

Theorem 39. Let $X$ be a tropical torus and $L=L(Q, \alpha)$ be a tropical line bundle on $X$ such that $Q=c_{1}(L)$ is positive semidefinite.

(1) If $\alpha$ lies in $\operatorname{Im}\left(q_{\mathbb{R}}\right)+\left(\mathbb{Z}^{n}\right)^{*}$, then $\Gamma(X, L)$ is generated by $l=\mid$ the torsion part of $\operatorname{Cok}(q) \mid$ elements as the $\mathbb{T}$-module and $\Gamma(X, L)$ embeds into $\mathbb{T}^{l}$ as in Definition 30. Moreover, $\Gamma(X, L)$ is identified with an $l$-dimensional convex polyhedron in $\mathbb{T}^{l}$ and its projectivization is identified with a compact polyhedron in $\mathbb{R}^{n}$.

(2) If $\alpha$ is not in $\operatorname{Im}\left(q_{\mathbb{R}}\right)+\left(\mathbb{Z}^{n}\right)^{*}$, then $\Gamma(X, L)=\{-\infty\}$.

\subsection{The rank of divisor}

In this section, we show that $h^{0}(X, D)$ introduced in Cartwright [6] is equal to the topological dimension of $\Gamma(X, \mathcal{O}(D))$.

Definition 40. Let $X$ be a tropical manifold. A divisor $D$ on $X$ is effective if the divisor $D$ is given by a global regular section of $\mathcal{O}(D)$. For a divisor $D$ on $X$, we denote the set of effective divisor linearly equivalent to $D$ by $|D|$. 
Definition 41 (Cartwright [6], Definition 3.1). Let $X$ be a tropical manifold and $D$ be a divisor on $X$. We define $h^{0}(X, D)$ as

$$
h^{0}(X, D):=\min \left\{\begin{array}{l|l}
k \in \mathbb{Z}_{\geq 0} \mid \begin{array}{l}
\text { there exist } k \text { points } p_{1}, \ldots, p_{k}, \text { such that } \\
\text { there is no divisor } E \in|D| \text { which passes all } p_{i}
\end{array}
\end{array}\right\} .
$$

If there is no such $k$, then we define $h^{0}(X, D)$ as $\infty$.

Remark 42. If $|D|=\emptyset$, then $h^{0}(X, D)=0$ holds trivially.

Remark 43. For a tropical curve $C$, we can check that $h^{0}(C, D)=r(D)+1$.

Let $X=\mathbb{R}^{n} / \Lambda$ be a topical torus and let $D$ be a divisor on $X$. By Proposition 28, $\mathcal{O}(D)$ is isomorphic to $L(Q, \alpha)$ for $Q=c_{1}(\mathcal{O}(D))$ and some $\alpha \in\left(\mathbb{R}^{n}\right)^{*}$. Let $q: \Lambda \rightarrow\left(\mathbb{Z}^{n}\right)^{*}$ be the linear map given by $Q$ and let $B=\left\{b_{1}, \ldots, b_{l}\right\} \subset\left(\mathbb{Z}^{n}\right)^{*}$ be a complete set of representatives of the torsion part of $\left(\mathbb{Z}^{n}\right)^{*} / q(\Lambda)$. We take the basis $\left\{\Theta_{b}\right\}_{b \in B}$ of $\Gamma(X, \mathcal{O}(D))$ defined in Section 3.3.

Theorem 44. The value $h^{0}(X, D)$ coincides with the topological dimension of $\Gamma(X, \mathcal{O}(D))$ as a convex polyhedron.

Proof. When the topological dimension of $\Gamma(X, \mathcal{O}(D))$ as a convex polyhedron is 0 or 1 , it is clear that $h^{0}(X, D)$ coincides with this dimension.

We assume that $\Gamma(X, \mathcal{O}(D)) \neq-\infty$ and $l \geq 2$. In order to see that $h^{0}(X, D) \geq l$, we take $l-1$-points $p_{1}, \ldots, p_{l-1} \in X$ and choose $q_{1}, \ldots, q_{l-1} \in \mathbb{R}^{n}$ such that $p_{i}=q_{i}+\Lambda$.

The tropical determinant $\operatorname{det}_{\text {trop }}\left(t_{i, j}\right)$ of a square matrix $\left(t_{i j}\right)_{1 \leq i, j \leq l}$ whose entries are in $\mathbb{T}$ is defined as $\max _{\sigma \in \mathfrak{S}_{l}}\left\{\sum_{i=1}^{n} a_{\sigma(i) i}\right\}$, where $\mathfrak{S}_{l}$ is the symmetric group of $\{1, \ldots, l\}$.

Now we define a theta function for $\mathcal{O}(D)$ by the Vandermonde determinant

$$
\begin{aligned}
V_{D}\left(x ; q_{1}, \ldots, q_{l-1}\right) & :=\operatorname{det}_{\text {trop }}\left(\begin{array}{cccc}
\Theta_{b_{1}}(x) & \Theta_{b_{2}}(x) & \ldots & \Theta_{b_{l}}(x) \\
\Theta_{b_{1}}\left(q_{1}\right) & \Theta_{b_{2}}\left(q_{1}\right) & \cdots & \Theta_{b_{l}}\left(q_{1}\right) \\
\vdots & \vdots & \ddots & \vdots \\
\Theta_{b_{1}}\left(q_{l-1}\right) & \Theta_{b_{2}}\left(q_{l-1}\right) & \cdots & \Theta_{b_{l}}\left(q_{l-1}\right)
\end{array}\right) \\
& =\max _{\sigma \in \mathfrak{S}_{l}}\left\{\Theta_{b_{\sigma(1)}}(x)+\sum_{i=2}^{l} \Theta_{b_{\sigma(i)}}\left(q_{i-1}\right)\right\} .
\end{aligned}
$$

We can see that the divisor on $X$ given by $V_{D}\left(x ; q_{1}, \ldots, q_{l-1}\right)$ is linearly equivalent to $D$ and passes the $l-1$ points $p_{1}, \ldots, p_{l-1}$. Thus we get $h^{0}(X, D) \geq l$.

Next, we suppose $h^{0}(X, D) \geq l+1$. Then for any $l$ points $p_{1}, \ldots, p_{l} \in X$, we can take a theta function $\Theta:=\max _{b \in B}\left\{\Theta_{b}(x)+t_{b}\right\}$ which is non-linear at a representative $q_{k} \in \mathbb{R}^{n}$ of $p_{k}$ for all $k=1, \ldots, l$. We set $H:=\bigcup_{b \in B} \operatorname{supp}\left(\operatorname{div} \Theta_{b}\right) \subset$ $\mathbb{R}^{n}$. We can assume that $q_{1}, \ldots, q_{l}$ are not in $H$.

Now we define a multigraph whose vertex set is $B$. For each $k=1, \ldots, l$, we can choose two distinct elements $b_{i_{k}}, b_{j_{k}} \in B$ such that $\Theta_{b_{i_{k}}}\left(q_{k}\right)+t_{b_{i_{k}}}=$ 
$\Theta_{b_{j_{k}}}\left(q_{k}\right)+t_{b_{j_{k}}}$ since $\Theta$ is singular at $q_{k}$. We connect $b_{i_{k}}$ and $b_{j_{k}}$ by an edge for each $k=1, \ldots, l$. Then we have a multigraph $\Gamma$ with $l$ vertices and $l$ edges. Such a $\Gamma$ necessarily has a cycle.

Let $b_{1}, \ldots, b_{k}$ make a cycle. We can assume that $b_{i} \neq b_{j}$ for $i \neq j$. Then we get

$$
\begin{aligned}
\Theta_{b_{1}}\left(q_{i_{1}}\right)+t_{b_{1}} & =\Theta_{b_{2}}\left(q_{i_{1}}\right)+t_{b_{2}} \\
\Theta_{b_{2}}\left(q_{i_{2}}\right)+t_{b_{2}} & =\Theta_{b_{3}}\left(q_{i_{2}}\right)+t_{b_{3}} \\
\vdots & \\
\Theta_{b_{k-1}}\left(q_{i_{k-1}}\right)+t_{b_{k-1}} & =\Theta_{b_{k}}\left(q_{i_{k-1}}\right)+t_{b_{k}} \\
\Theta_{b_{k}}\left(q_{i_{k}}\right)+t_{b_{k}} & =\Theta_{b_{1}}\left(q_{i_{k}}\right)+t_{b_{1}}
\end{aligned}
$$

for some $q_{i_{1}}, \ldots q_{i_{k}}$. By combining these equations, we get

$$
\left(\Theta_{b_{1}}\left(q_{i_{1}}\right)-\Theta_{b_{2}}\left(q_{i_{1}}\right)\right)+\cdots+\left(\Theta_{b_{k-1}}\left(q_{i_{k-1}}\right)-\Theta_{b_{k}}\left(q_{i_{k-1}}\right)\right)+\left(\Theta_{b_{k}}\left(q_{i_{k}}\right)-\Theta_{b_{1}}\left(q_{i_{k}}\right)\right)=0 .
$$

More generally, we define the functions $l_{i_{1}, \ldots, i_{k}}$ for $k>1$ and a subset $\left\{i_{1}, \ldots, i_{k}\right\} \subset$ $\{1, \ldots, l\}$ as follows:

$$
l_{i_{1}, \ldots, i_{k}}\left(x_{1}, \ldots x_{k}\right):=\sum_{j=1}^{k}\left(\Theta_{b_{i_{j}}}\left(x_{j}\right)-\Theta_{b_{i_{j+1}}}\left(x_{j}\right)\right),
$$

where $i_{k+1}:=i_{1}$. These functions are rational functions on $\left(\mathbb{R}^{n}\right)^{k}$. To summarize the above argument, we obtain the following lemma.

Lemma 45. Suppose $h^{0}(X, D) \geq l+1$. Then for any $l$ points $q_{1} \ldots, q_{l} \in$ $\mathbb{R}^{n} \backslash H$, there exist an ordered sequence $\left\{i_{1}, \ldots, i_{k}\right\}$ of elements in $\{1, \ldots, l\}$ and $\left\{q_{j_{1}}, \ldots, q_{j_{k}}\right\} \subset\left\{q_{1} \ldots, q_{l}\right\}$ such that $l_{i_{1}, \ldots, i_{k}}\left(q_{j_{1}}, \ldots q_{j_{k}}\right)=0$.

We define subsets $H_{1}, H_{k} \subset\left(\mathbb{R}^{n}\right)^{l}, k=2, \ldots, l$ as below.

$$
\begin{gathered}
H_{1}:=\left\{\left(x_{1}, \ldots, x_{l}\right) \in\left(\mathbb{R}^{n}\right)^{l} \mid x_{i} \in H \text { for some } i\right\} . \\
H_{k}:=\left\{\left(x_{1}, \ldots, x_{l}\right) \in\left(\mathbb{R}^{n}\right)^{l} \mid \begin{array}{c}
\text { There exist an ordered sequence }\left\{i_{1}, \ldots, i_{k}\right\} \text { of elements in }\{1, \ldots, l\} \\
\text { and a subset }\left\{x_{j_{1}}, \ldots, x_{j_{k}}\right\} \subset\left\{x_{1}, \ldots, x_{l}\right\}, \\
\text { such that } l_{i_{1}, \ldots, i_{k}}\left(x_{j_{1}}, \ldots x_{j_{k}}\right)=0
\end{array}\right\} .
\end{gathered}
$$

It is clear that the dimension of $H_{1}$ is $l n-1$. The dimension of $H_{k}$, for $k=2, \ldots, l$ is also $l n-1$ since functions $l_{i_{1}, \ldots, i_{k}}$ are rational functions on $\left(\mathbb{R}^{n}\right)^{l}$ with non-zero slopes. Thus $\mathbb{R}^{l n} \backslash \cup_{k=1}^{l} H_{k}$ is not empty and we can take $l$ points $q_{1}, \ldots, q_{l}$ with $\left(q_{1}, \ldots, q_{l}\right) \in\left(\mathbb{R}^{n}\right)^{l} \backslash \cup_{k=1}^{l} H_{k}$. By definition, for any subset $\left\{q_{j_{1}}, \ldots q_{j_{k}}\right\}$ of these $l$ points and any ordered sequence $\left\{i_{1}, \ldots, i_{k}\right\}$ of $\{1, \ldots, l\}, l_{i_{1}, \ldots, i_{k}}\left(q_{j_{1}}, \ldots q_{j_{k}}\right) \neq 0$. This contradicts Lemma 45 . Thus we get $h^{0}(X, D)<l+1$. 


\section{Riemann-Roch inequality for tropical abelian varieties}

\subsection{Intersection number of divisors on a tropical torus}

In this section, we briefly define the intersection number of $n$ divisors on an $n$-dimensional tropical torus. For the details, we refer the reader to AllermannRau [1] and Mikhalkin [15].

Let $C_{1}, C_{2}, \ldots, C_{n}$ be divisors in $\mathbb{R}^{n}$. We take rational polyhedral subdivisions of $C_{1}, \ldots, C_{n}$ such that the intersection $\bigcap_{i=1}^{n} C_{i}$ is a rational polyhedral subcomplex of each $C_{i}$. We define $S$ to be the set of vertices of $\bigcap_{i=1}^{n} C_{i}$.

At first, we define the intersection multiplicity at a point $p \in S$. For each $i=1, \ldots, n$, we can take an open neighborhood $U_{i}$ of $p$ in $C_{i}$ which is contained in the open star of $p$, so that $p$ is the only vertex of $\bigcap_{i=1}^{n} U_{i}$. Let $U_{i}\left(v_{i}\right)$ be the translation of $U_{i}$ by a vector $v_{i} \in \mathbb{R}^{n}$. For generic sufficiently small $v_{i}, \bigcap_{i=1}^{n} U_{i}\left(v_{i}\right)=\emptyset$ or $U_{1}\left(v_{1}\right), \ldots, U_{n}\left(v_{n}\right)$ intersect transversally and none of intersection points lie in $(n-2)$-dimensional cells of $U_{i}\left(v_{i}\right)$.

If $\bigcap_{i=1}^{n} U_{i}\left(v_{i}\right)=\emptyset$, we define the intersection multiplicity at $p$ to be 0 . If $\bigcap_{i=1}^{n} U_{i}\left(v_{i}\right) \neq \emptyset$, for every intersection point $q \in \bigcap_{i=1}^{n} U_{i}\left(v_{i}\right)$, there exist (n-1)dimensional cells $E_{i}$ of $U_{i}\left(v_{i}\right)$ such that $\{q\}=\bigcap_{i=1}^{n} E_{i}$. Let $w_{i}$ be the weight of $E_{i}$ and $v_{i}$ be a primitive vector orthogonal to $E_{i}$. We define the intersection multiplicity at $q$ as $\operatorname{mult}(q):=w_{1} w_{2} \cdots w_{n}\left|\operatorname{det}\left(v_{1}, v_{2}, \ldots, v_{n}\right)\right|$ and we define the intersection multiplicity at a point $p \in S$ to be $\operatorname{mult}(p):=\sum_{q \in \bigcap_{i=1}^{n} U_{i}\left(v_{i}\right)} \operatorname{mult}(q)$. We can check that this definition is independent of the choice of $U_{1}, \ldots, U_{n}$ and generic sufficiently small vectors $v_{i}$ because of the balancing condition of divisors. For more details, we refer the reader to [1], Construction 6.4] or [15, Definition 4.4].

Now we can define the intersection number $C_{1} . C_{2} \ldots C_{n}$ to be $\sum_{p \in S} \operatorname{mult}(p)$ if the set $S$ is finite. This definition is independent of the subdivision. If we take finer subdivisions of $C_{1}, \ldots, C_{n}$, a new point of $S$ is on the interior of a cell of $\bigcap_{i=1}^{n} C_{i}$ and its intersection multiplicity is 0 .

Let $X$ be an $n$-dimensional tropical torus and $D_{1}, D_{2}, \ldots, D_{n}$ be divisors on $X$. We take rational polyhedral subdivisions of $D_{1}, \ldots, D_{n}$ as above and define $S$ to be the set of vertices of $\bigcap_{i=1}^{n} D_{i}$, which is finite set because of the compactness of $X$. Since $X$ locally looks like $\mathbb{R}^{n}$, we can define the intersection multiplicities of vertices of $S$ in the same way as above. The intersection number of $D_{1}, D_{2}, \ldots, D_{n}$ is defined to be the sum of the intersection multiplicities of vertices of $\mathrm{S}$.

It is clear that the intersection number is an $n$-linear form on $\operatorname{Div}(X)$. Moreover, it is invariant under linear equivalence, that is,

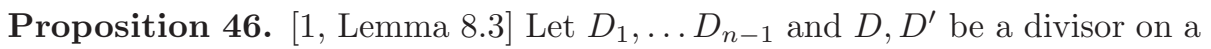
tropical torus $X / \Lambda$. If $D$ and $D^{\prime}$ are linearly equivalent, then $D_{1} \ldots D_{n-1} \cdot D=$ $D_{1} \ldots D_{n-1} \cdot D^{\prime}$.

Proof. We take a rational function $f: \mathbb{R}^{n} / \Lambda \rightarrow \mathbb{R}$ such that $D(f)=D-D^{\prime}$ and define a rational function $f_{t}$ on $\mathbb{R}^{n} / \Lambda$ for $t \in \mathbb{R}$ to be $\max \{t, f\}$. Since the 
divisor $D\left(f_{t}\right)$ deforms continuously, the intersection number $D_{1} \ldots D_{n-1} \cdot D\left(f_{t}\right)$ is continuous for $t$, in particular, it is constant for $t$.

Let $t_{-}, t_{+}$be a real number such that $f_{t_{-}}=t_{-}, f_{t_{+}}=f$. Then

$$
\begin{aligned}
D_{1} \ldots D_{n-1} \cdot D(f) & =D_{1} \ldots D_{n-1} \cdot D\left(f_{t_{+}}\right) \\
& =D_{1} \ldots D_{n-1} \cdot D\left(f_{t_{-}}\right) \\
& =D_{1} \ldots D_{n-1} \cdot 0=0 .
\end{aligned}
$$

\subsection{Self-intersection number of divisors}

For a symmetric form $Q \in \Lambda^{*} \otimes\left(\mathbb{Z}^{n}\right)^{*}$, we $\operatorname{define} \operatorname{det} Q$ as the determinant of the matrix representation of $Q$ with respect to a basis of $\left(\mathbb{Z}^{n}\right)^{*}$ and a basis of $\Lambda^{*}$ which define the same orientation of $\left(\mathbb{R}^{n}\right)^{*}$.

Theorem 47. Let $X=\mathbb{R}^{n} / \Lambda$ be a tropical abelian variety and $D$ be a divisor on $\mathrm{X}$. Then $\frac{1}{n !} D^{n}=\operatorname{det} c_{1}(\mathcal{O}(D))$. In particular, $\frac{1}{n !} D^{n}$ is always an integer.

Proof. Firstly, we calculate the self-intersection number of a divisor $D$ for the line bundle $L\left(E_{n}, 0\right)$ on the tropical torus $\mathbb{R}^{n} / \mathbb{Z}^{n}$. We can assume that the divisor $D$ is a parallel translate of $\bigcup_{i=1}^{n}\left\{\left(x_{1}, \ldots, x_{n}\right) \in \mathbb{R}^{n} \mid x_{i} \in \mathbb{Z}\right\}$ with weight 1. To calculate the self-intersection number $D^{n}$, we consider the intersection number $\left(D+\varepsilon e_{1}\right) \cdot\left(D+\varepsilon e_{2}\right) \ldots .\left(D+\varepsilon e_{n}\right)$, where $e_{1}, \ldots, e_{n} \in \mathbb{Z}^{n}$ is the standard basis and $\varepsilon$ is a sufficiently small positive number. Then we can see that an intersection points of $\left(D+\varepsilon e_{1}\right), \ldots,\left(D+\varepsilon e_{n-1}\right)$, and $\left(D+\varepsilon e_{n}\right)$ corresponds to a permutation of numbers $\{1, \ldots, n\}$. Since the intersection multiplicity of each intersection point is 1 , we get $D^{n}=n !$. Figure 6 is a 2 -dimensional example of these calculations

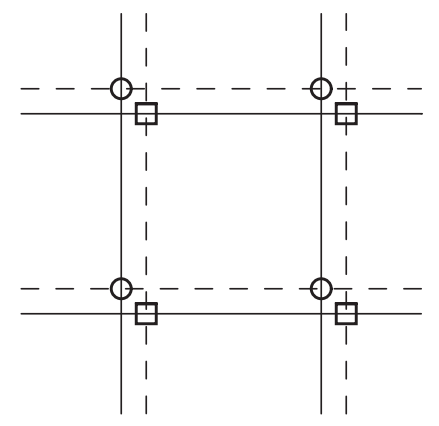

Figure 6: Calculating the self-intersection number of the divisor associated to $L\left(E_{2}, 0\right)$

Second, we consider a divisor $D$ for a line bundle $L(Q, 0)$ where $Q$ is positive definite and principal. We consider a family of symmetric matrices $Q_{s}:=((1-$ $\left.s) E_{n}+s Q^{-1}\right)^{-1}$ and a family of lattices $\Lambda_{s}=q_{s}^{-1}\left(\mathbb{Z}^{n}\right)^{*}$ for $s \in(-\varepsilon, 1+\varepsilon)$. Here 
$q_{s}^{-1}:\left(\mathbb{Z}^{n}\right)^{*} \rightarrow \Lambda_{s} \subset \mathbb{R}^{n}$ is the linear map induced by $Q_{s}^{-1}$ and $\varepsilon$ is a sufficiently small number such that $Q_{s}$ can be defined.

We define a $\left(\mathbb{Z}^{n}\right)^{*}$-action on $\mathbb{R}^{n} \times(-\varepsilon, 1+\varepsilon)$ by

$$
a \cdot(x, s):=\left(Q_{s}^{-1} \cdot a+x, s\right), a \in\left(\mathbb{Z}^{n}\right)^{*},(x, s) \in \mathbb{R}^{n} \times(-\varepsilon, 1+\varepsilon) .
$$

We define $\mathscr{X}$ to be the quotient of $\left(\mathbb{R}^{n} \times(-\varepsilon, 1+\varepsilon)\right)$ by the $\left(\mathbb{Z}^{n}\right)^{*}$-action and $X_{s}:=\left(\mathbb{R}^{n} \times\{s\}\right) /\left(\mathbb{Z}^{n}\right)^{*}=\mathbb{R}^{n} / \Lambda_{s} . \mathscr{X}$ is a tropical manifold and $X_{s}$ is a divisor of $\mathscr{X}$.

Let $\Theta(x, s)$ be a regular function of $\mathbb{R}^{n} \times(-\varepsilon, 1+\varepsilon)$ defined to be

$$
\begin{aligned}
\Theta(x, s) & :=\max _{\lambda \in q_{s}^{-1}\left(\left(\mathbb{Z}^{n}\right)^{*}\right)}\left\{Q_{s}(\lambda, x)-\frac{1}{2} Q_{s}(\lambda, \lambda)\right\} \\
& =\max _{a \in\left(\mathbb{Z}^{n}\right)^{*}}\left\{a \cdot x-\frac{1}{2} Q_{s}\left(q_{s}^{-1}(a), q_{s}^{-1}(a)\right)\right\} \\
& =\max _{a \in\left(\mathbb{Z}^{n}\right)^{*}}\left\{a \cdot x-\frac{1}{2} Q_{s}^{-1}(a, a)\right\} .
\end{aligned}
$$

Then $\Theta(x, s)$ satisfies the following quasi-periodicity;

$$
\begin{aligned}
\Theta\left(x+q_{s}^{-1}(a), s\right) & =\max _{a^{\prime} \in\left(\mathbb{Z}^{n}\right)^{*}}\left\{a^{\prime} \cdot\left(x+q_{s}^{-1}(a)\right)-\frac{1}{2} Q_{s}^{-1}\left(a^{\prime}, a^{\prime}\right)\right\} \\
& =\max _{a^{\prime} \in\left(\mathbb{Z}^{n}\right)^{*}}\left\{a^{\prime} \cdot x+Q_{s}^{-1}\left(a^{\prime}, a\right)-\frac{1}{2} Q_{s}^{-1}\left(a^{\prime}, a^{\prime}\right)\right\} \\
& =\max _{a^{\prime} \in\left(\mathbb{Z}^{n}\right)^{*}}\left\{a^{\prime} \cdot x-\frac{1}{2} Q_{s}^{-1}\left(a^{\prime}-a, a^{\prime}-a\right)+\frac{1}{2} Q_{s}^{-1}(a, a)\right\} \\
& =\max _{a^{\prime} \in\left(\mathbb{Z}^{n}\right)^{*}}\left\{\left(a^{\prime}+a\right) \cdot x-\frac{1}{2} Q_{s}^{-1}\left(a^{\prime}, a^{\prime}\right)+\frac{1}{2} Q_{s}^{-1}(a, a)\right\} \\
& =\max _{a^{\prime} \in\left(\mathbb{Z}^{n}\right)^{*}}\left\{a^{\prime} \cdot x-\frac{1}{2} Q_{s}^{-1}\left(a^{\prime}, a^{\prime}\right)\right\}+a \cdot x+\frac{1}{2} Q_{s}^{-1}(a, a) \\
& =\Theta(x, s)+a \cdot x+\frac{1}{2} Q_{t}^{-1}(a, a)
\end{aligned}
$$

Thus $\Theta(x, s)$ gives a divisor $[\Theta(x, s)]$ on $\mathscr{X}$.

Since the intersection number $[\Theta(x, s)]^{n} . X_{t}$ is constant for $t$, we get

$$
D^{n}=[\Theta(x, s)]^{n} \cdot X_{1}=[\Theta(x, s)]^{n} \cdot X_{0}=n ! .
$$

Next, we consider the case where $Q$ is positive definite but not necessary principal. Let $\Xi$ be the section of $\mathcal{O}(D)$ satisfying $\varphi(\Xi)=0$ and let $\widetilde{\Lambda} \subset \Lambda \otimes \mathbb{R}$ be the lattice $q_{\mathbb{R}}^{-1}\left(\left(\mathbb{Z}^{n}\right)^{*}\right)$, which is an overlattice of $\Lambda$. Then $\Xi$ satisfies the quasi-periodicity

$$
\Xi(x+\eta)=\Xi(x)+Q_{\mathbb{R}}(\eta, x)+\frac{1}{2} Q_{\mathbb{R}}(\eta, \eta)+Q_{\mathbb{R}}(\eta, r), \eta \in \widetilde{\Lambda}, x \in \mathbb{R}^{n}
$$


and thus the divisor $[\Xi]$ is $\widetilde{\Lambda}$-periodic, while $\left[\Theta_{b}\right]$ is $\Lambda$-periodic. Since the selfintersection number of $[\Xi]$ on $\mathbb{R}^{n} / \widetilde{\Lambda}$ is $n$ !, the self-intersection number of $\left[\Theta_{b}\right]$ on $\mathbb{R}^{n} / \Lambda$ is $n ! \cdot|\widetilde{\Lambda} / \Lambda|=n ! \operatorname{det} Q$.

Finally, we show the statement for a divisor $D$ which is not necessary positive definite. Let $\mathcal{L}$ be the abelian group $\operatorname{Im} c_{1} \times\left(\mathbb{R}^{n}\right)^{*}$ and $\mathcal{L}_{\mathbb{R}}$ be the linear space $\left(\operatorname{Im} c_{1} \otimes \mathbb{R}\right) \times\left(\mathbb{R}^{n}\right)^{*}$. An element $(Q, \alpha)$ of $\mathcal{L}$ gives rise to a line bundle $L(Q, \alpha)$.

Since $X$ is a tropical abelian variety, a line bundle $L(Q, \alpha)$ on $X$ has a rational section. We define an $n$-linear form $\eta$ on $\mathcal{L}^{n}$ to be $\eta\left(\left(Q_{1}, \alpha_{1}\right), \ldots,\left(Q_{n}, \alpha_{n}\right)\right)=$ $\frac{1}{n !}\left(D_{1} \ldots D_{n}\right)$. Here $D_{i}$ is a divisor on $X$ given by a rational section of $L\left(Q_{i}, \alpha_{i}\right)$. This $\eta$ is well-defined because the intersection number depends only on the linear equivalence class of divisors. Let $\eta_{\mathbb{R}}: \mathcal{L}_{\mathbb{R}}^{n} \rightarrow \mathbb{R}$ be the natural extension of $\eta$. Then $\eta_{\mathbb{R}}((Q, \alpha), \ldots,(Q, \alpha))=\operatorname{det} Q$ when $Q$ is positive definite.

That is, $\eta_{\mathbb{R}}((Q, \alpha), \ldots,(Q, \alpha))$ and $\operatorname{det} Q$ coincide on an intersection of $\mathcal{L}$ and an open cone in $\mathcal{L}_{\mathbb{R}}$. Since both of them are polynomials of degree $n$, they must coincide on the whole space $\mathcal{L}_{\mathbb{R}}$. Therefore $\frac{1}{n !} D^{n}=\operatorname{det} c_{1}(\mathcal{O}(D)) \in \mathbb{Z}$ for all divisor $D$ on $X$.

Corollary 48. Let $X$ be a tropical abelian surface and $D$ be a divisor on $X$. Then the Riemann-Roch inequality

$$
h^{0}(X, D)+h^{0}(X,-D) \geq \frac{1}{2} D^{2} .
$$

holds. More precisely, when we write $Q=c_{1}(\mathcal{O}(D))$ and $\mathcal{O}(D)=L(Q, \alpha)$,

(1) If $Q$ is positive or negative definite,

$$
h^{0}(X, D)+h^{0}(X,-D)=\frac{1}{2} D^{2}=\operatorname{det} Q .
$$

(2) If $Q$ is positive or negative semidefinite and $Q$ has 0 as an eigenvalue,

- If $Q \neq 0$ and $\alpha$ lies in $\operatorname{Im} q_{\mathbb{R}}+\left(\mathbb{Z}^{2}\right)^{*}$,

$\mid$ the torsion part of $\operatorname{Cok}(q) \mid=h^{0}(X, D)+h^{0}(X,-D)>\frac{1}{2} D^{2}=0$,

where $q: \Lambda \rightarrow\left(\mathbb{Z}^{n}\right)^{*}$ is the linear map given by $Q=c_{1}(\mathcal{O}(D))$ and $q_{\mathbb{R}}: \mathbb{R}^{n} \rightarrow\left(\mathbb{R}^{n}\right)^{*}$ is the extension of $q$.

- If $Q=0$ and $\alpha$ lies in $\left(\mathbb{Z}^{2}\right)^{*}$,

$$
2=h^{0}(X, D)+h^{0}(X,-D)>\frac{1}{2} D^{2}=0,
$$

- If $\alpha$ does not lie in $\operatorname{Im} q_{\mathbb{R}}+\left(\mathbb{Z}^{2}\right)^{*}$,

$$
h^{0}(X, D)+h^{0}(X,-D)=\frac{1}{2} D^{2}=0 .
$$


(3) If $Q$ is indefinite,

$$
0=h^{0}(X, D)+h^{0}(X,-D)>\frac{1}{2} D^{2}=\operatorname{det} Q .
$$

Proof. The values of right-hand side was calculated in the above theorem. On the other hand, we get the value of left-hand side by combining Theorem 14 and Theorem 39 .

Remark 49. The above result gives us a desirable value of $h^{1}(X, D)$, although we don't have any reasonable definition of it.

Remark 50. These consequences are compatible with the Riemann-Roch inequality for classical abelian varieties. See 国 Corollary 3.5.4, Theorem 3.6.1 and 3.6.3].

\section{Acknowledgements}

I am deeply grateful to my advisor Hiroshi Iritani for his advice. This paper would not have been possible without his guidance.

Special thanks go to Yuji Odaka for his helpful advice. In particular, he suggested to study the Riemann-Roch inequality for surfaces with trivial canonical class.

I would like to thank Dustin Cartwright for his valuable comments. He informed me of his definition of $h^{0}(X, D)$ and suggested to study the relationship between $h^{0}(X, D)$ and $\operatorname{dim} H^{0}(X, \mathcal{O}(D))$, and also to study the integrality of $\frac{1}{n !} D^{n}$. Theorem 44 and Theorem 47 were then obtained.

\section{References}

[1] Allermann, L., Rau, L.: First steps in tropical intersection theory, Mathematische Zeitschrift, 264.3, 633-670 (2010)

[2] Allermann, L.: Chern classes of tropical vector bundles, Arkiv for Matematik, 50.2, 237-258 (2012)

[3] Baker, M., Norine, S.: Riemann-Roch and Abel-Jacobi theory on a finite graph, Advances in Mathematics, 215.2, 766-788 (2007)

[4] Birkenhake, C., Lange, H.: Complex Abelian Varieties, 2nd ed., Springer Grundlehren,.302 (2004)

[5] Cartwright, D.: Combinatorial tropical surfaces, arXiv preprint, arXiv1506.02023 (2015)

[6] Cartwright, D.: A specialization inequality for tropical complexes. arXiv preprint, arXiv 1511.00650 (2015) 
[7] Chan, M.: Combinatorics of the tropical Torelli map, Algebra and Number Theory, 6.6, 1133-1169 (2012)

[8] Gathmann, A., Kerber, M.: A Riemann-Roch theorem in tropical geometry, Mathematische Zeitschrift, 259.1, 217-230 (2008)

[9] Gathmann, A., Kerber, M., Markwig,H.: Tropical fans and the moduli spaces of tropical curves, Compositio Mathematica, 145.1, 173-195 (2009)

[10] Gross, M.: Tropical geometry and mirror symmetry, Journal of the American Mathematical Society, 114 (2011)

[11] Haase, C., Musiker, G., Yu, J.: Linear systems on tropical curves, Mathematische Zeitschrift, 270.3, 1111-1140 (2012)

[12] Jell, P., Rau, J., Shaw, K.: Lefschetz $(1,1)$-theorem in tropical geometry, arXiv preprint, arXiv:1711.07900 (2017).

[13] Kristin, S.: Tropical surfaces, arXiv preprint, arXiv:1506.07407 (2015)

[14] Mikhalkin, G.: Enumerative tropical algebraic geometry in $\mathbb{R}^{2}$, Journal of the American Mathematical Society, 18.2, 313-377 (2005)

[15] Mikhalkin, G.: Tropical geometry and its applications, Proceedings of the International.Congress of Mathematicians, 2, 827-852 (2006)

[16] Mikhalkin, G., Zharkov, I.: Tropical curves, their Jacobians and theta functions, Curves and abelian varieties, 465, 203-230 (2008)

[17] Mikhalkin, G., Zharkov, I.: Tropical eigenwave and intermediate Jacobians, Homological mirror symmetry and tropical geometry, Springer International Publishing, 309-349 (2014) 